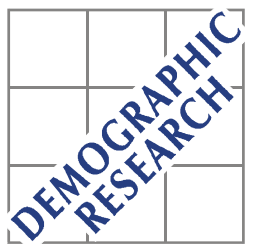

Demographic Research a free, expedited, online journal of peer-reviewed research and commentary in the population sciences published by the Max Planck Institute for Demographic Research Doberaner Strasse 114 D D-18057 Rostock · GERMANY www.demographic-research.org

DEMOGRAPHIC RESEARCH

VOLUME 6, ARTICLE 6, PAGES 91-144

PUBLISHED 01 MARCH 2002

www.demographic-research.org/Volumes/Vol6/6/

DOI: $10.4054 /$ DemRes.2002.6.6

\title{
Tempo-Adjusted Period Parity \\ Progression Measures, Fertility \\ Postponement and Completed Cohort \\ Fertility
}

Hans-Peter Kohler

José Antonio Ortega

(C) 2002 Max-Planck-Gesellschaft. 


\section{Table of Contents}

1 Introduction 92

$2 \quad$ Revisiting the Implications of Postponed 93

$\begin{array}{ll} & \text { Childbearing on Period and Cohort Fertility } \\ 2.1 \quad \text { Intuitive introduction to tempo-adjusted period } & 93\end{array}$

$\begin{array}{ll}\text { parity progression measures } \\ 2.2 & \text { Description of period fertility and comparison with }\end{array}$

$\begin{array}{ll}\text { adjusted } T F R & \\ 2.3 \quad \text { Continued fertility postponement and cohort } & 99\end{array}$ fertility

3 Adjusting Parity Progression Rates for Tempo and 102

Variance Effects

$\begin{array}{lll}3.1 & \text { A parity and age model of fertility } & 102\end{array}$

$\begin{array}{lll}3.2 & \text { Tempo distortions in childbearing intensities } & 103\end{array}$

$\begin{array}{ll}3.3 & \text { Measuring and adjusting for tempo changes } \\ \end{array}$

4 Parity Progression Measures under Alternative 112

4.1 Extrapolation of fertility postponement to future 112

$\begin{array}{lll} & \text { periods } & 117\end{array}$

$\begin{array}{lll}4.3 & \text { Conditional parity progression ratios } & 118\end{array}$

$\begin{array}{lll}4.4 & \text { Conditional cohort mean age at birth } & 121\end{array}$

$5 \quad$ Using the Model: Measuring Period Fertility and 124

Completing Cohort Fertility

$\begin{array}{lll}5.1 & \text { Parity progression measures for measuring period } & 124\end{array}$

5.2 Completing the fertility of cohorts 125

$\begin{array}{lll}6 & \text { Empirical Implementation } & 126\end{array}$

$\begin{array}{ll}\text { Notes } & 129\end{array}$

$\begin{array}{ll}\text { References } & 132\end{array}$

$\begin{array}{lll}\text { A } & \text { Appendix } & 137\end{array}$ 


\title{
Tempo-Adjusted Period Parity Progression Measures, Fertility Postponement and Completed Cohort Fertility
}

\author{
Hans-Peter Kohler ${ }^{1} \quad$ José Antonio Ortega ${ }^{2}$
}

\begin{abstract}
In this paper we introduce a new set of tempo-adjusted period parity progression measures in order to account for two distinct implications caused by delays in childbearing: tempo distortions imply an underestimation of the quantum of fertility in observed period data, and the fertility aging effect reduces higher parity births because the respective exposure is shifted to older ages when the probability of having another child is quite low. Our measures remove the former distortion and provide means to assess the latter aging effect. The measures therefore provide a unified toolkit of fertility indices that $(a)$ facilitate the description and analysis of past period fertility trends in terms of synthetic cohort measures, and $(b)$ allow the projection of the timing, level and distribution of cohort fertility conditional on a specific postponement scenario. Due to their explicit relation to cohort behavior, these measures extend and improve the existing adjustment of the total fertility rate. We apply these methods to Sweden from 1970 to 1999.
\end{abstract}

\footnotetext{
${ }^{1}$ Head of Research Group on Social Dynamics and Fertility, Max Planck Institute for Demographic Research, Doberaner Str. 114, 18057 Rostock, Germany. Tel: +49-381-2081-123, Fax: +49-381-2081-423, Email: kohler@demogr.mpg.de, www: http://user.demogr.mpg.de/kohler.

${ }^{2}$ Departamento de Análisis Económico: Economía Cuantitativa, Universidad Autónoma de Madrid, 28049Madrid, Spain, Email: joseantonio.ortega@uam.es, www: http://www.adi.uam.es/ jaortega.
} 


\section{Introduction}

Substantial fluctuations in the level of period fertility are often coupled with marked changes in the timing of births. For instance, the postponement of fertility has importantly contributed to the emergence of low and lowest-low fertility in Europe during the 1990s (e.g., Kohler, Billari, and Ortega 2001). These changes in the timing of fertility are of central relevance for demographers interested in the measurement and projection of fertility. On the one hand, future postponement patterns have important implications for cohort fertility and the recuperation of delayed childbearing. On the other hand, past and current delays of childbearing affect the properties and interpretation of standard period fertility measures. In particular, a postponement or anticipation of fertility renders the level of standard period fertility measures different from the level that would have been observed in the absence of tempo changes. These differences are called tempo distortions, and the period fertility level in the absence of tempo distortions is called tempo-adjusted fertility or quantum.

Despite the widespread agreement about the relevance of such tempo distortions, the appropriate way of measuring and adjusting for these effects remains controversial. Bongaarts and Feeney (1998, henceforth BF) have proposed an adjusted total fertility rate that measures the total fertility rate that would have been observed in a calendar year if there had been no change in the tempo of fertility during that year. Kohler and Philipov (2001, henceforth KP) have extended this adjustment to include variance effects, i.e., changes in the shape of the fertility schedule in addition to shifts in the mean age. Alternative models for adjusting the $T F R$ have also been proposed (e.g., Brass 1990; Le Bras 1997), and virtually all of the above approaches are strongly influenced by the seminal work of Norman Ryder (e.g., Ryder 1964, 1983) (see also the review of the literature on period fertility measures in Ortega and Kohler 2002a).

In addition to the debate about the appropriate adjustment for tempo distortions, there is also a controversy about the relation between tempo-adjusted fertility measures and cohort fertility. For instance, while the adjusted $T F R$ in the Bongaarts-Feeney and Kohler-Philipov analysis provides an improved indicator of period fertility (or quantum), it cannot be used to infer the completed fertility of the current cohorts of women. This limitation is due to the fact that the completed cohort fertility depends on the future paths of fertility quantum and tempo. It can only be properly projected with appropriate assumptions about both aspects of future fertility trends. Moreover, investigating these implications of future quantum and tempo changes on cohort fertility requires an explicit consideration of the fact that only women who are currently at parity zero, one, two, etc., are exposed to giving birth to their first, second, third, etc., child. This sequencing of births is not explicitly considered in the calculation of the adjusted $T F R$ or the standard $T F R$. In many circumstances, therefore, these measures provide little—or even erroneous- 
information about the parity distribution and the completed fertility of cohorts (Kim and Schoen 2000; Ortega and Kohler 2002b; van Imhoff 2001).

In order to overcome this limitation, we develop in this paper tempo-adjusted period parity progression measures that are based on an explicit parity and age fertility model with a long tradition in demographic research (e.g., Park 1976; Quensel 1939; Rallu and Toulemon 1994; Whelpton 1946). These tempo-adjusted parity progression measures provide a new and unified 'tool-kit' that can be used for two related purposes. First, they remove tempo distortions and parity composition effects from the observed period fertility pattern and therefore provide an improved indicator of the period quantum of fertility. In particular, our tempo-adjusted period parity progression measures suggest a natural synthetic-cohort measure of period fertility and quantum, denoted period fertility index, that is equal to the total fertility of women who experience the tempo-adjusted period childbearing intensities (or adjusted age-specific parity progression probabilities) during their life-course. Second, our measures allow a demographically correct and consistent projection of the level, timing and distribution of the completed fertility of cohorts who have not finished childbearing, conditional on the future paths of quantum and tempo. We derive these assumptions about future quantum and tempo from observed period fertility patterns. Our methods therefore provide an important input for population projections, and future analyses can combine these methods with stochastic forecasting models that explicitly consider the uncertainty about the future development of quantum and tempo. Moreover, the close relationship between period fertility measurement and cohort projection reduces the traditional distinction between 'cohort' and 'period' approaches to fertility (e.g., Ní Bhrolcháin 1992): our methods provide proper period measures of fertility that can be combined with projection scenarios in order to assess the cohort fertility that results from period fertility patterns.

\section{Revisiting the Implications of Postponed Childbearing on Period and Cohort Fertility}

\subsection{Intuitive introduction to tempo-adjusted period parity progres- sion measures}

We begin our paper with an intuitive discussion of our methods. The aim of this introductory discussion is to convey the main concepts and ideas of our analysis in a non-technical manner. The formal development follows in Sections 3 to 5 .

Our discussion in this section is based on the fertility trend in Sweden during the last decades, which has been of great interest to demographers for its distinct and unusual pattern (for a more comprehensive discussion, see Kohler and Ortega 2002). Whereas 
fertility levels stagnated or declined in many European countries during the 1980s, Sweden experienced a baby boom after 1985. Between 1985 and 1990 the TFR increased from 1.74 to a level of 2.13, exceeding the replacement level. In the 1990s this baby boom was displaced by an equally swift baby bust, and by 1998 the total fertility rate had declined to an historically low level of 1.51 (Council of Europe 1999). Three questions are of central importance in this context. First, how does the description of the Swedish fertility trends change from the analysis based on the adjusted $T F R$ to the analysis based on our Period Fertility Index $(P F)$ that reflects synthetic cohort fertility? Second, what inferences can be made about the completed fertility and the final parity distribution of cohorts who have not finished childbearing as of 1999, on the basis of the most recent fertility patterns? Third, how would a potential future postponement of fertility, which mirrors the most recent postponement patterns observed in the 1990s, change the fertility level and parity distribution attained by cohorts?

All three of the above questions can be answered with the measures proposed in this paper. The data required for these analyses include the births by age and order in a calendar year and a measure of the person-years lived by women who are 'at risk' of giving birth to a first, second, third, etc., child. The former information is identical to the data requirements for the adjustment of the total fertility rate. The latter information is more specific. For instance, the exposure can be estimated by the mid-year female population by age and parity. In countries with population registers it can be obtained from the exact counts of the person-years lived by age and parity during a calendar year. The primary advantage of these additional data is that they allow us to base our analyses on occurrence-exposure rates, or childbearing intensities, that reflect the age-specific 'hazard' of experiencing another birth for women at some specific parity. In our specific case, these childbearing intensities are calculated on the basis of Andersson's (1999) data that include annual births by parity and age in Sweden and estimates of the corresponding person-years of exposure in all calendar years from 1970-1999 (see also Andersson and Guiping 2001). These data are restricted to Swedish women and do not include foreignborn women living in Sweden.

Childbearing intensities — and also age-specific parity progression probabilities-have long been used to calculate period parity progression measures (Hoem and Jensen 1982; Rallu and Toulemon 1994; Schoen 1988). Unfortunately, this standard calculation remains affected by tempo distortions. In particular, a fertility postponement distorts the parity progression ratios through two important pathways. On the one hand, tempo distortions lead to an underestimation of the probability that a woman in the synthetic cohort experiences another birth conditional on her current age and parity. On the other hand, the presence of a fertility postponement delays the age at which women are exposed to the risk of higher parity births. This can potentially lead to a reduction of the progression to higher parities, which we denote as the fertility aging effect associated with a postpone- 
ment of childbearing. This effect can be partially or totally compensated for if the fertility schedule at higher parities is shifted as a response to the postponement at lower parities. In this case, we speak of a net fertility aging effect.

There is substantial micro-evidence that the compensation for delays in entering parenthood is not complete, and several recent analyses have shown that higher age at first birth is causally associated with a lower completed fertility level (Billari and Kohler 2002; Kohler, Skytthe, and Christensen 2001; Morgan and Rindfuss 1999). This evidence is obtained from a variety of studies including Danish twin data and survey data from Italy, Spain, Bulgaria, the Czech Republic, Hungary and Sweden; it is also consistent with earlier micro-studies on the relationship between the timing and level of fertility (e.g., Bumpass and Mburugu 1977; Bumpass et al. 1978; Marini and Hodsdon 1981; Presser 1971; Trussell and Menken 1978). When considered together, these studies leave few doubts that cohort fertility patterns are characterized by a net fertility aging effect. Despite the pervasive micro-evidence, however, fertility aging effects have not been carefully investigated in aggregate analyses of contemporary fertility patterns. Our new measures overcome this limitation and provide a possibility of studying the implications of fertility aging with aggregate data.

We base our analysis on a parity and age model of fertility that can lead to net fertility aging effects when a delay of first (or second, third,...) births is not compensated for by a postponement at higher parities. [Note 1] In our subsequent analyses we formalize this parity and age model, and we implement our tempo-adjusted period parity progression measures via the following steps: First, we transfer the KP framework, which was initially developed for the adjustment of the total fertility rate, to childbearing intensities. This allows us to estimate adjusted childbearing intensities that are not distorted by tempo effects. As in KP, our definition of tempo-change operates at the age-specific level and therefore incorporates potential age-period interactions: there can be a differential pace of fertility postponement at different ages, and there can even be a postponement at some ages and an anticipation of fertility at other ages (see KP for further discussions).

Second, we use the adjusted childbearing intensities as building blocks for period fertility measures and projection methods for cohort fertility. Specifically:

(a) Adjusted childbearing intensities can be used within a synthetic cohort model to measure and describe period fertility based on a variety of tempo-adjusted parity progression measures, including: (i) the period lifetime birth probability of $n$ additional children that is equal to the probability that a woman, who experiences the tempo-adjusted period intensities during her life-course, gives birth to at least $n$ additional children; and (ii) the period fertility index $(P F)$ that is equal to completed fertility of women who experience the tempo-adjusted period childbearing intensities. This period fertility index can be regarded as a proper measure of the quantum of fertility. 
(b) Adjusted childbearing intensities can also be used for projection purposes to study the fertility of cohorts who are still in childbearing ages, conditional on scenarios about the future quantum and tempo of period fertility. In particular, our methods provide a tool to investigate dependence of cohort fertility on the pace of fertility postponement and/or recuperation during the life-course of a cohort. For simplicity, we concentrate in this paper on two particular benchmark scenarios: (i) a postponement stops scenario in which we calculate the parity progression measures assuming that the postponement comes to a halt after the reference year, i.e., assuming that there is no further delay of childbearing in future periods; and (ii) a postponement continues scenario in which we assume that the tempo change observed in a reference year prevails during the life-course of the cohort for which fertility is projected.

In addition to improved period measurement and cohort projections, our tempo-adjusted parity progression measures derived from period childbearing intensities also imply an important advantage regarding empirical measurement of tempo changes. In particular, childbearing intensities constitute occurrence-exposure rates that reflect the 'risk' of an additional child conditional on parity and age. These intensities therefore constitute demographic rates of type 1 (taux de première catégorie; see Henry 1972). They differ from standard parity- and age-specific fertility incidence rates or demographic rates of type 2 (taux de deuxième catégorie; see Lotka and Spiegelman 1940), which are obtained at each age by dividing births of a given order by the female population irrespective of parity. Unfortunately, this lack of disaggregation in the denominator of incidence rates can lead to severe distortions in the inferences of the level and particularly the timing of fertility from period data. These distortions occur because the population distribution by parity fluctuates over time, and these fluctuations induce changes in both the level and age-pattern of period incidence rates (Feeney and Lutz 1991; Ortega and Kohler 2002b; Rallu and Toulemon 1994; van Imhoff 2001). Changes in the population parity composition can therefore suggest tempo- and quantum changes in analyses of period incidence rates, even if there have been no changes in an individual's parity-specific fertility behavior (for a detailed discussion, see Ortega and Kohler 2002b). These compositional influences are avoided if the analyses are directly based on childbearing intensities that constitute occurrence-exposure rates of fertility.

\subsection{Description of period fertility and comparison with adjusted $T F R$}

The application of our measures to the description of period fertility patterns is illustrated in Figure 1 that compares our parity progression based measures with the standard and adjusted total fertility rate.

Figure 1(a) shows the observed total fertility rate for first births, the corresponding adjusted $T F R$ for first births and the period lifetime probability of having at least one child 
Figure 1: Left-hand graph: comparison of total fertility rate, adjusted $T F R$ and period lifetime birth probability for first births with and without tempo-adjustment. Right-hand graph: comparison of total fertility rate, adjusted $T F R$ (all birth orders), period fertility index (PF) and the index of total fertility (TF) in the postponement continues scenario.

(a) First births: TFR and PPRs

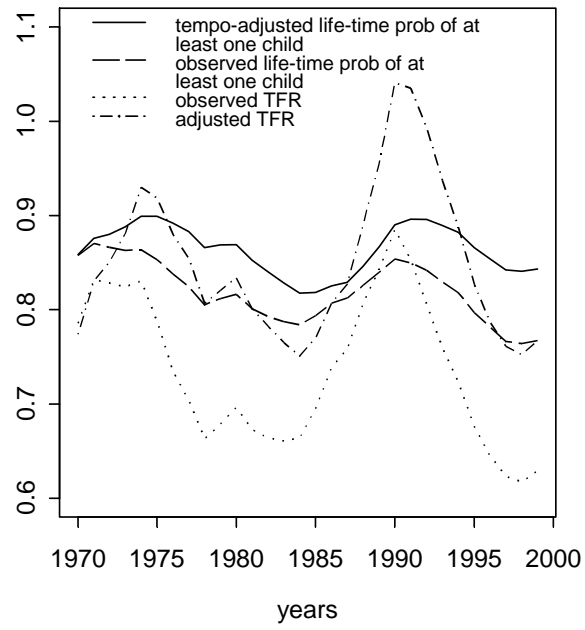

(b) All parities: TFR and PF

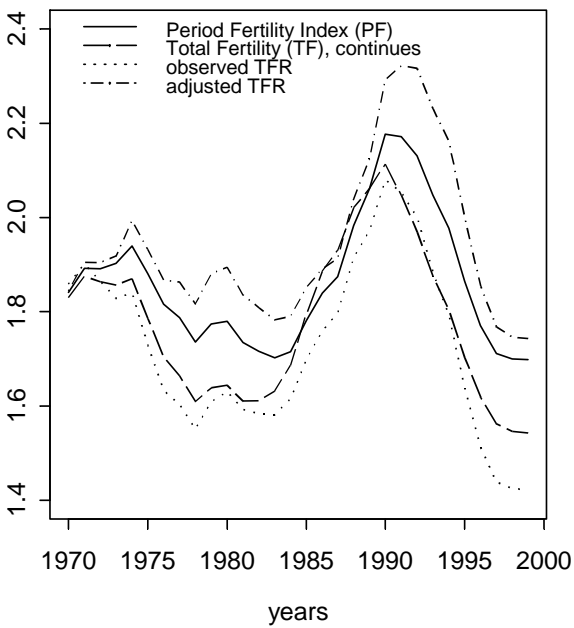

(or equivalently, the progression probability from parity zero to parity one). The latter is depicted with and without an adjustment for tempo-distortions. All four measures are indicators of the level of fertility for first births, and they fluctuate between 1970 and 1999 in accordance with the overall trend in total fertility: an initial increase is followed by a decline and recovery during the baby boom; another decline occurs after 1990. Despite this common overall pattern, however, there are important conceptual and empirical differences among these measures. First, the adjusted $T F R$ and the tempo-adjusted lifetime probability of at least one child attempt to correct for tempo distortions in the observed period pattern. The adjusted $T F R$ and the adjusted lifetime birth probability, therefore, exceed the corresponding measures obtained from the observed fertility pattern without tempo-adjustment. This underestimation of first-birth fertility from the observed pattern is greatest in periods with the most rapid delays in entering parenthood around 1980 and in the 1990s. Second, the measures reflect quite different trends during the period from 1970 to 1999 . For instance, the total fertility rate for first births and the adjusted $T F R$ 
for first births exhibit large fluctuations over time. In comparison to these large fluctuations, the period lifetime probability of having at least one child is relatively constant. This is particularly the case after tempo-distortions are removed, and the tempo-adjusted lifetime birth probability neither increases nor decreases as pronouncedly as the standard or adjusted total fertility rate for first births. On the one hand, these different developments are partially due to the fact that changes in the parity distribution of a population subsequent to economic changes or policy interventions can cause fluctuations in period fertility measures that are unrelated to behavioral changes in that specific period. Since Sweden experienced varying socioeconomic conditions and family-policies during the period under investigation (Andersson 1999, 2000; Hoem 2000; Hoem and Hoem 1996; Hoem 1990), the total fertility rate for first births therefore overestimates the volatility of fertility behavior (for related analyses see Ortega and Kohler 2002b; van Imhoff 2001). On the other hand, the differences between the measures in Figure 1(a) are partially due to the fact that neither the standard nor the adjusted total fertility rate for first births represent synthetic cohort measures. Neither measure can thus properly reflect the number of first-births that occur to members of a synthetic cohort based on the level of first-birth fertility in a given period.

Both of the above limitations are avoided using a parity progression based indicator of period fertility, and these measures therefore yield a different-and in our opinion improved-picture of fertility trends for first births. In particular, the tempo-adjusted period lifetime probability of at least one child does not fluctuate substantially during the observation period, and it does not substantially decline in the baby-bust period after 1990.

While the level of first birth fertility has been relatively constant during the baby boom and bust after 1985 in Sweden, this is not the case for the period fertility index $(P F)$ that exhibits substantial fluctuations from 1970 to 1999 (Figure 1b). This index, which equals the complete fertility of women experiencing the tempo-adjusted period childbearing intensities, increases from less than 1.8 in the early 1980 s to more than 2.1 in 1990 and subsequently declines again to about 1.7 . Since tempo-adjusted lifetime birth probabilities for the first child have remained relatively constant, this analysis implies that the baby boom and bust is primarily due to fluctuations in the parity progression ratios to the second and third child (for related studies, see Andersson 1999, 2000; Hoem 2000; Hoem and Hoem 1996; Hoem 1990; Kohler and Ortega 2002).

Figure 1(b) also allows a comparison of $T F R$-based fertility measures with the measures proposed in this paper. The most important insight from this comparison is that the fertility attained by the synthetic cohort can deviate substantially from the level suggested by the adjusted $T F R$, which is due to the compositional distortions in the TFR and in the inference of tempo changes (see also Kim and Schoen 2000; van Imhoff and Keilman 2000). In addition, the parity progression measures in Figure 1(b) reflect how the 
differential tempo changes at different parities potentially affect completed fertility. For most periods, the changes in the tempo of fertility tend to be faster for lower parity births and slower for higher parity births. This suggests the presence of a net fertility aging effect so that a continued postponement of fertility tends to reduce the completed fertility. This reduction of total fertility in the postponement continues scenario, as compared to the period fertility index, reaches its highest levels based on the patterns observed around 1980 and in the 1990s. Only in the late 1980s do the differences diminish or even reverse, which is due to the fact that the delayed onset of parenthood is compensated for by sufficiently fast tempo changes at second birth. Since a continued delay of childbearing for the foreseeable future seems more likely than an imminent leveling off in the pace of fertility postponement, the period fertility index, which assumes no further postponement, can be regarded as an upper bound for the completed cohort fertility implied by the fertility pattern of a given reference year.

\subsection{Continued fertility postponement and cohort fertility}

In addition to the measurement of period fertility, our tempo-adjusted parity progression measures are also attractive because they provide a direct application to the projection of cohort fertility. In Figure 2(a), for instance, we consider the cohorts who are 17 and 24 years old in 1999, and we project the proportion of women who will still be childless in the years $2005,2010, \ldots$ under the assumption that the 1999 parity-specific fertility levels prevail in the future. These calculations are augmented in Figure 2(b) with the projected level of final childlessness in all cohorts who are still in childbearing ages in 1999.

The full line in these two figures reflects the transition into parenthood that is obtained from the observed childbearing intensities, and these calculations project an ultimate level of childlessness around $23 \%$. However, because the pace of fertility postponement has been quite high during the 1990s in Sweden, this projection is distorted by tempo effects and does not reflect the true cohort experience that is implied by the 1999 level of fertility. The unbiased calculations based on the adjusted childbearing intensities with no further postponement of fertility (dashed lines) project a substantially more rapid transition into parenthood and a substantially lower level of ultimate childlessness of about $15 \%$. Hence, projections based on the observed childbearing intensities, which are tempo-distorted in periods of a fertility postponement, tend to underestimate the fraction of women who are going to experience at least one child given the current level of fertility.

This ultimate level of childlessness in cohorts does not depend on assumptions about future postponement patterns. [Note 2] However, important differences exist between the postponement stops and the postponement continues scenario with respect to the timing of entering parenthood. If the postponement of fertility is assumed to continue at the pace observed in 1999, the transition into parenthood is delayed (Figure 2a and Table 1). This 
Figure 2: Projection of fertility behavior for cohorts who have not finished childbearing in 1999 based on the level of fertility and postponement pattern observed in 1999

(a) Proportion still childless at time $\mathrm{t}$

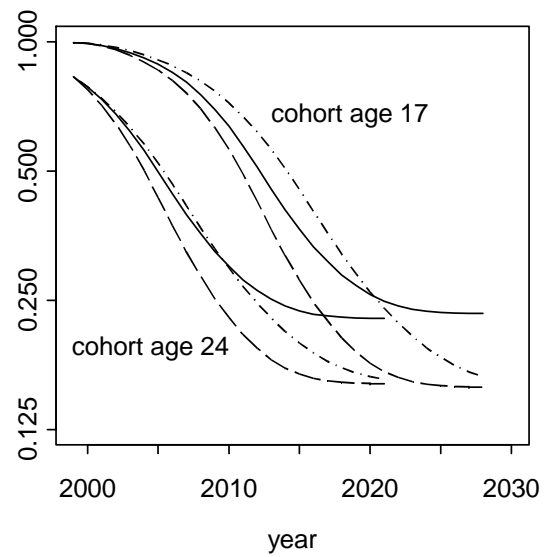

(c) Cumulative cohort fertility

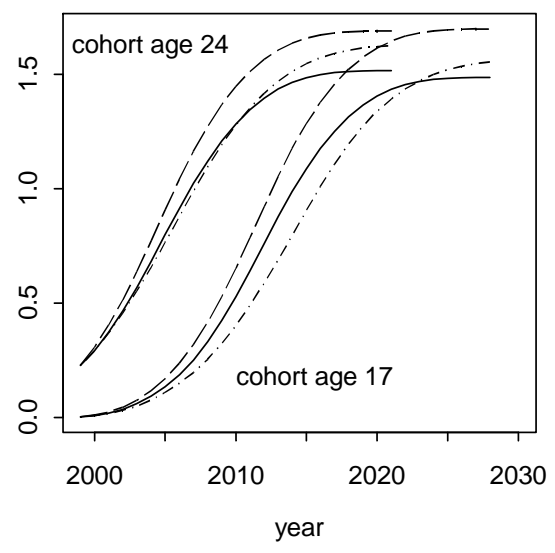

(b) Prop. remaining ultimately childless

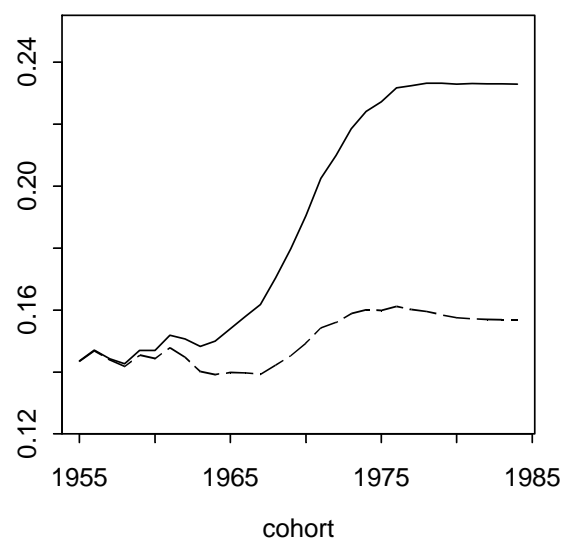

(d) Completed fertility

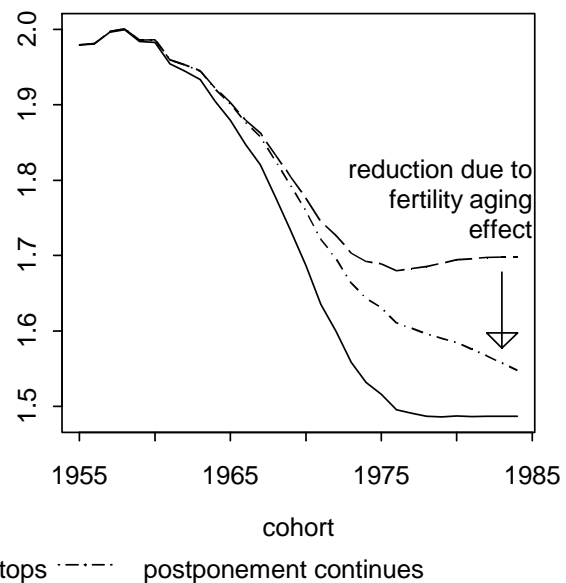

Notes: The postponement stops (dashed line) and postponement continues (dashed-dotted line) are based on the tempo-adjusted fertility intensities and assume either no further delays in childbearing or a further delay in childbearing that mirrors the 1999 postponement pattern. In Graph $(b)$ these two postponement scenarios imply identical results and are not distinguished from each other. The full line in all graphs indicates the projection obtained from the observed fertility intensities. 
Table 1: Projected mean age at birth for women in the cohorts born 1982 (age 17 in 1999) and born 1975 (age 24 in 1999)

\begin{tabular}{|c|c|c|}
\hline & $\begin{array}{c}\text { Cohort age } 17 \\
\text { in } 1999\end{array}$ & $\begin{array}{c}\text { Cohort age } 24 \\
\text { in } 1999\end{array}$ \\
\hline \multicolumn{3}{|l|}{ Mean age at first birth } \\
\hline postponement stops scenario & 28.34 & $28.56^{a}$ \\
\hline postponement continues scenario & 31.56 & 30.03 \\
\hline calculated from observed data & 28.86 & 28.79 \\
\hline \multicolumn{3}{|l|}{ Mean age at second birth } \\
\hline postponement stops scenario & 31.05 & $31.18^{a}$ \\
\hline postponement continues scenario & 33.76 & 32.44 \\
\hline calculated from observed data & 31.42 & 30.30 \\
\hline \multicolumn{3}{|l|}{ Mean age at birth, all parities } \\
\hline postponement stops scenario & 30.01 & $30.16^{a}$ \\
\hline postponement continues scenario & 32.57 & 31.27 \\
\hline calculated from observed data & 30.37 & 30.27 \\
\hline
\end{tabular}

effect of a continued postponement on the transition into parenthood is more pronounced in the younger cohort because the postponement continues for a prolonged time until this cohort reaches the primary ages of childbearing.

In Figure 2(c) we shift our analysis to the combined fertility across all birth orders and report the cumulative cohort fertility for women who are age 17 and 24 in 1999. In both cohorts an ongoing postponement of fertility implies a lower cumulative fertility in all years after 1999 as compared to the postponement stops scenario. This pattern is due to two factors. On one hand, the entry into parenthood is postponed towards older ages. On the other hand, the delayed onset of parenthood shifts the beginning of being 'at risk' of a second and higher order births to ages (see Table 1), and at these relatively old ages the probability of experiencing a second or third birth declines rapidly. Corresponding calculations from observed childbearing intensities suggest a cumulative fertility that is below the postponement stops scenario, and this difference is due to the tempo distortions in the observed period intensities. However, the relation is no longer as clear-cut if the postponement of fertility is assumed to continue. In particular, the observed intensities and the postponement continues scenario imply an approximately equal cumulative fertility until about 2010 in the cohort who is age 24 in 1999, and afterwards a contin- 
ued postponement suggests a higher cumulative fertility due to some late first and higher order births. In the cohort that is age 17 in 1999, the postponement continues scenario implies a cumulative fertility level until about 2020 that is substantially below the level suggested by the observed intensities. Due to relatively late childbearing the completed fertility in this cohort will exceed the completed fertility suggested by the observed data, but it will fall substantially short of the level attained by the cohort in the postponement stops scenario. [Note 3] This pattern, which is illustrated for the cohorts who are 17 and 24 years old in 1999, pertains similarly to all cohorts who have not completed fertility as of 1999 (Figure 2d). While the completed fertility of cohorts starts to stabilize at a level of about 1.7 for cohorts who are below age 25-30 in 1999 under the postponement stops scenario, cohort fertility continues to decline in young cohorts if the postponement of fertility continues at the pace observed in 1999. These further declines in the postponement continues scenario are due to a net fertility aging effect: the number of higher order births is reduced by an ongoing delay of childbearing because women tend to be 'at risk' of second and higher order births only at ages when the probability of experiencing second, third, and fourth births is already quite low. [Note 4]

\section{Adjusting Parity Progression Rates for Tempo and Vari- ance Effects}

\subsection{A parity and age model of fertility}

In this section we begin our formal development of the tempo-adjusted period parity progression measures that we introduced in the previous application to Sweden. As we have pointed out in the previous sections, we develop these measures within a parity and age fertility model that relies on the following assumption:

Assumption 1 Determinants of fertility: Fertility behavior in the population depends only on age, period and parity, and it is independent of the timing of any previous births.

Given this assumption, multi-state population theory (Hoem and Jensen 1982; Schoen 1988) provides the tools for studying the family building process based on the 'hazard' of experiencing a birth of a given parity at different reproductive ages. In our model, this 'hazard' is given by the parity-and age-specific childbearing intensity at time t for a women age a at parity $j$, denoted as $m_{j}(a, t)$. It can be calculated by dividing the order$(j+1)$ births occurring to women at age $a$ by the person years lived in a calendar year by women who are age $a$ and parity $j$. Formally the age-specific childbearing intensities are 
defined as

$$
\begin{gathered}
m_{j}(a, t)=\lim _{\Delta t \rightarrow 0} \operatorname{Pr}(\text { birth of order } j+1 \text { birth occurs between time } t \\
\text { and } t+\Delta t \mid \text { age } a, \text { time } t, \text { parity } j) / \Delta t,
\end{gathered}
$$

where the conditional probability in Eq. (1) is the probability that a woman who is age $a$ and parity $j$ at time $t$ experiences a birth of order $j+1$ before time $t+\Delta t$. [Note 5]

Childbearing intensities can be easily converted into parity progression probabilities. For instance, the probability that a woman age $x$ and parity $j$ at time $T$ experiences at least one additional birth prior to age $y$, denoted $p_{j}^{T}(x, y)$, follows from the integration of the childbearing intensities $m_{j}(a, t)$ in Eq. (1) along the cohort line in the Lexis diagram as

$$
p_{j}^{T}(x, y)=1-\exp \left[-\int_{x}^{y} m_{j}(a, T+(a-x)) d a\right] .
$$

\subsection{Tempo distortions in childbearing intensities}

In order to analyze tempo distortions in childbearing intensities and derived period parity measures, we also need to specify how changes in the tempo and level of fertility affect the observed childbearing intensities. For this purpose we utilize the assumption that age, parity and period are the only determinants of fertility (Assumption 1), and we separate the observed childbearing intensities into two factors: first, a level effect, $q_{j}(t)$, that proportionally increases the parity- $j$ childbearing intensities at all ages; and second, an age-pattern of childbearing intensities, $h_{j}(a, t)$, that evolves over time only due to the postponement of fertility (an analog assumption also underlies the adjustment of the total fertility rate in $\mathrm{BF}$ and $\mathrm{KP})$.

Assumption 2 Observed childbearing intensities: The observed intensity schedule for parity $j$ women can be decomposed as

$$
m_{j}(a, t)=q_{j}(t) \cdot h_{j}(a, t),
$$

where the term $q_{j}(t)$ is a parity-and period-specific level effect that proportionally increases or decreases childbearing intensities at all ages, and $h_{j}(a, t)$ is a schedule that determines the age-pattern of the parity- $j$ childbearing intensities at time $t$.

Our model therefore assumes a very general definition of level effects that proportionally increases the parity- $j$ childbearing intensities at all ages. Any influences on fertility that do not affect childbearing intensities proportionally at all ages alter the shape of the intensity schedule and introduce a tempo-change. Moreover, the schedule $h_{j}(a, t)$ changes 
over time only due to changes in the timing of fertility at parity $j$, and it remains constant in periods when there is no postponement or anticipation of fertility at any age. The characterization of fertility postponement in our analyses can therefore be based exclusively on the evolution of the age-pattern $h_{j}(a, t)$ over time.

In order to provide a benchmark for measuring changes in the timing of fertility, we choose an arbitrary reference year $T$. We then specify a hypothetical intensity schedule $\phi_{j}^{T}(a)$ that reflects the age-pattern of childbearing intensities that would prevail after the reference year $T$ in the absence of changes in the tempo of fertility subsequent to the reference year $T$ :

Assumption 3 Age-pattern of childbearing intensities in absence of tempo changes: If tempo changes are absent at all time periods $t \geq T$, the age-pattern of childbearing intensities at parity $j$ does not change over time and is given by a standardized schedule $\phi_{j}^{T}(a)$. We assume for simplicity that the integral of this standardized schedule equals unity, i.e., we assume that $\int \phi_{j}^{T}(a) d a=1$.

In general, the age-pattern of the intensity schedule $\phi_{j}^{T}(a)$ is unknown and needs to be identified and estimated on the basis of the observed age-pattern $h_{j}(a, t)$ and its evolution over time. In order to solve this inverse estimation problem, our analysis needs to establish the formal relation between the observed age-pattern of childbearing intensities, $h_{j}(a, t)$, and the hypothetical age-pattern, $\phi_{j}^{T}(a)$. Once this relation is established within our model, the evolution of observed age-pattern $h_{j}(a, t)$ over time can be used to estimate both the extent of tempo changes and the unobserved age-pattern $\phi_{j}^{T}(a)$ in the reference year $T$ (see Section 3.3).

We introduce the cumulated tempo change $R_{j}^{T}(a, t)$ as the primary indicator of tempo changes. In a formal sense, the cumulated tempo change is merely a function that allows a transformation of the age-pattern of childbearing intensities over time; its interpretation in terms of fertility postponement will become apparent after our formal definition of fertility postponement below. Specifically, we will show that the cumulative tempo increases with age in periods when births are delayed and decreases with age (and possibly becomes negative) when births are anticipated. Moreover, with the exception of some technical restrictions, the cumulated tempo $R_{j}^{T}(a, t)$ can be specified in a quite arbitrary manner. Our analyses can therefore capture very general postponement patterns that vary across age, cohort and period. (Note: In the following, we use the notation $\Delta_{a}$ and $\Delta_{t}$ to denote partial derivatives of a function with respect to age $a$ and time $t$, and we use $\Delta_{a, t}$ as a shortcut to denote the aging operator $\left.\left(\Delta_{a}+\Delta_{t}\right)=\frac{\partial}{\partial a}+\frac{\partial}{\partial t}\right)$ :

Assumption 4 Cumulated tempo change: Denote as $R_{j}^{T}(a, t)$ a smooth function that represents the cumulated tempo changes that have occurred between the reference year $T$ 
and time $t$ for women of parity $j$. We require that $R_{j}^{T}(a, T)=0$ and $\left.\Delta_{a} R_{j}^{T}(a, t)\right|_{t=T}=0$ at all ages $a$, and additionally we require that $a-R_{j}^{T}(a, t)$ is increasing in a. [Note 6]

This definition merely specifies the formal properties of the function $R_{j}^{T}(a, t)$ and does not yet provide an interpretation of cumulated tempo changes in terms of changes in the timing of fertility. In the next step we therefore provide an explicit notion of fertility postponement (or delays in childbearing) that is defined and measured in terms of cumulated tempo changes $R_{j}^{T}(a, t)$ at each parity $j$. This definition of fertility postponement is based on a comparison of the observed age-pattern of childbearing intensities, $h_{j}(a, t)$, and the age-pattern $\phi_{j}^{T}(a)$ that would prevail at $t \geq T$ if there were no further postponement of fertility after the reference year $T$.

In order to facilitate this comparison, we define two hypothetical probabilities of experiencing a birth at parity $j$. These probabilities are based on the intensity schedules $h_{j}(a, t)$ and $\phi_{j}^{T}(a)$ respectively. The specification in terms of these intensity schedules implies that we consider a situation in which there are no changes in the parity-specific level effects; the postponement of fertility is the only process that affects childbearing intensities over time.

First, we consider a woman who is age $x$ and parity $j$ in the reference $T$, and who experiences the childbearing intensities $h_{j}(a, t)$. We then denote as $p_{j, T}^{h}\left(x, y^{\prime}\right)$ the probability of a birth of order $j+1$ to the above woman during the age interval $\left(x, y^{\prime}\right]$, or equivalently, during the time interval $\left(T, T^{\prime}\right]$ where $T^{\prime}=T+\left(y^{\prime}-x\right)$. This probability follows by integrating the childbearing intensities $h_{j}(a, t)$ along the cohort line in the Lexis diagram as

$$
p_{j, T}^{h}\left(x, y^{\prime}\right)=1-\exp \left[-\int_{x}^{y^{\prime}} h_{j}(a, T+(a-x)) d a\right] .
$$

We also define a second probability, denoted as $p_{j, T}^{\phi}\left(x, y^{\prime \prime}\right)$, that reflects the likelihood of a birth of order $j+1$ to the above woman in the case that there is no further postponement of childbearing after the reference year $T$. In this case, the age-pattern of childbearing intensities for all times $t \geq T$ is time-invariant and is given by $\phi_{j}^{T}(a)$ (see Assumption 3 ). The probability that the above woman experiences a birth of order $j+1$ during the age interval $\left(x, y^{\prime \prime}\right]$ on the basis of these intensities is obtained by integrating the intensity schedule $\phi_{j}^{T}(a)$ as

$$
\pi_{j, T}^{\phi}\left(x, y^{\prime \prime}\right)=1-\exp \left[-\int_{x}^{y^{\prime \prime}} \phi_{j}^{T}(a) d a\right] .
$$

The comparison of the above probabilities then provides a formal definition of fertility postponement: 
Definition 1 Postponement of fertility: A postponement of fertility at parity $j$ implies that for all ages $x$ and $y^{\prime}$ the probabilities $p_{j, T}^{h}\left(x, y^{\prime}\right)$ and $p_{j, T}^{\phi}\left(x, y^{\prime \prime}\right)$ satisfy

$$
p_{j, T}^{h}\left(x, y^{\prime}\right)=\pi_{j, T}^{\phi}\left(x, y^{\prime}-R_{j}^{T}\left(y^{\prime}, T+\left(y^{\prime}-x\right)\right)\right),
$$

that is, a postponement of fertility implies that $p_{j, T}^{h}\left(x, y^{\prime}\right)$ is equal to $p_{j, T}^{\phi}\left(x, y^{\prime \prime}\right)$ evaluated at $y^{\prime \prime}=y^{\prime}-R_{j}^{T}\left(y^{\prime}, T^{\prime}\right)$ with $T^{\prime}=T+\left(y^{\prime}-x\right)$.

For illustration, consider a cohort of women who are age $x$ and parity $j$ in the reference year $T$, and assume that the 'risk' of a birth at parity $j$ is given by the observed age-pattern of childbearing intensities $h_{j}(a, t)$. In the presence of a postponement, the fraction of women who experience a birth of order $j+1$ between age $x$ and $y^{\prime}$ (or equivalently, during the time period from $T$ to $T^{\prime}=T+\left(y^{\prime}-x\right)$ ) is given by $\pi_{j, T}^{h}\left(x, y^{\prime}\right)$. If there had been no postponement of fertility after the reference year $T$, then the same fraction of women would have experienced their births of order $j+1$ between age $x$ and $y^{\prime \prime}=$ $y^{\prime}-R_{j}^{T}\left(y^{\prime}, T^{\prime}\right)$, or equivalently during the time interval from $T$ to $T^{\prime}-R_{j}^{T}\left(y^{\prime}, T^{\prime}\right)$. These age and time intervals are shorter than the corresponding intervals $\left(x, y^{\prime}\right]$ and $\left(T, T^{\prime}\right]$ whenever the cumulative tempo $R_{j}^{T}\left(y^{\prime}, T^{\prime}\right)$ is positive; that is, whenever there has been a delay of childbearing over time.

The above definition therefore captures the essential element of the postponement of fertility: if there is no change in the level effect $q_{j}(t)$ over time, then delayed childbearing implies that age and time intervals are stretched without affecting the probability of giving birth during these intervals. This condition is reflected in the fact that the birth probabilities in the presence and absence of the postponement, $\pi_{j, T}^{h}\left(x, y^{\prime}\right)$ and $\pi_{j, T}^{\phi}\left(x, y^{\prime \prime}\right)$, are equal. The births at parity $j$ that occur during the age interval $\left(x, y^{\prime}\right]$ in the presence of a postponement, therefore, would have occurred in the age interval $\left(x, y^{\prime}-R_{j}^{T}\left(y^{\prime}, T^{\prime}\right)\right]$ in the absence of a postponement.

This relation is further illustrated by the Lexis diagrams in Figure 3. In this Lexis diagram, we have chosen the year 1990 as reference period $T$ for measuring the delay of childbearing. We then consider the births at parity $j$ that occur during the person-years of exposure that are indicated by the parallelogram $\mathrm{ABCD}$. That is, we consider the births of order $j+1$ that are observed during the period from 1990 to 1991 to the cohort of women who are age 30-31 and parity $j$ in 1990. We assume that the period 1990-91 has been characterized by a constant level effect $q_{j}(t)$ and a delay of childbearing at parity $j$.

If this delay of childbearing after 1990 had been absent, then all births in the parallelogram ABCD would have occurred in the area AEFD. The presence of a postponement between $t=1990$ and $t=1991$, therefore, extends the area AEFD to the larger parallelogram $\mathrm{ABCD}$. The same number of births occurs over a larger number of person-years, which in turn implies that the postponement of fertility leads to a downward distortion of childbearing intensities. 
Figure 3: Postponement of fertility in the Lexis diagram

(a) no age-period interactions

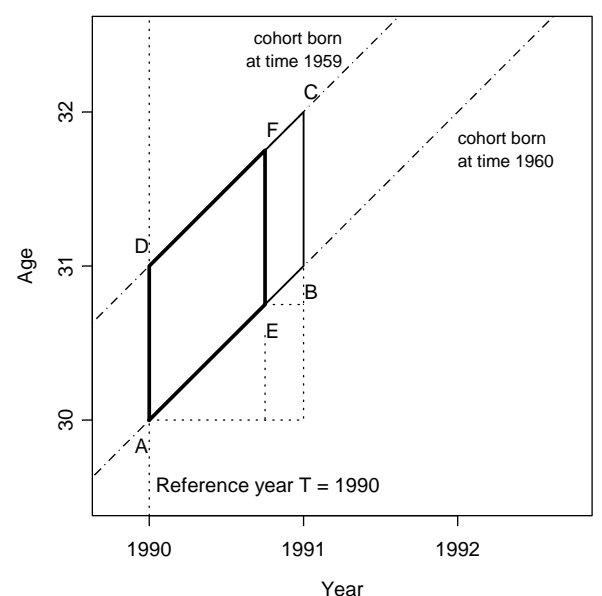

(b) with age-period interactions

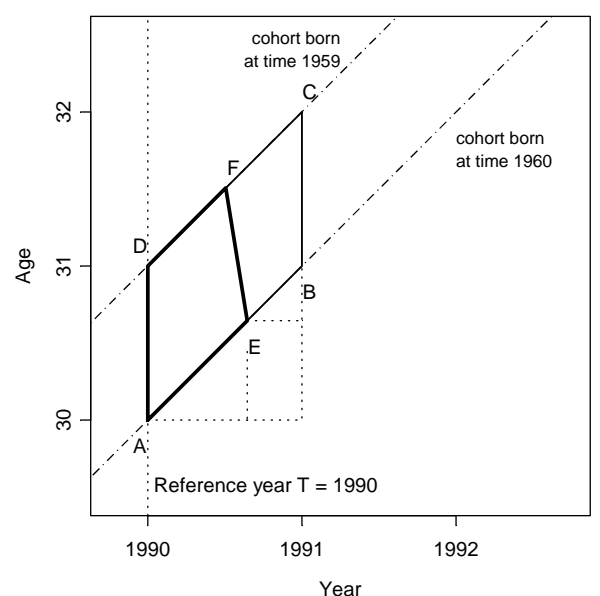

Notes: In the left-hand graph the cumulated tempo is a function of only time and not age, while in the right-hand graph the cumulated tempo depends on both time and age. In the former case, the initial parallelogram ABCD is transformed into another parallelogram AEFD. In the latter case, the transformation is more general and the area AEFD is no longer a parallelogram. Such age-period interactions in the postponement of fertility can lead to changes in the shape of the intensity schedule over time.

The extent of fertility postponement during the period 1990-91 in Figure 3 is reflected by the horizontal and vertical distances between right-side corners of the parallelograms $\mathrm{ABCD}$ and AEFD. In addition, this extent is measured by the cumulated tempo at time $t=1991$. For instance, the vertical and horizontal distance between the points B,E equals $R_{j}^{1990}(31,1991)$, i.e., it is equal to the additional postponement of fertility that has occurred during the period from $t=1990$ to $t=1991$ for women who are age 31 in 1991 and were age 30 and parity $j$ in 1990. Similarly, the vertical and horizontal distance between the points C,F is equal to $R_{j}^{1990}(32,1991)$, which reflects the additional postponement during the period 1990-91 for women who are age 32 in 1991 and were age 31 and parity $j$ in 1990. Births that occur at age 31 and time 1991 (see point B in Figure 3), therefore, would have occurred at time $1991-R_{j}^{1990}(31,1991)$ and at an age $31-R_{j}^{1990}(31,1991)$ if the fertility postponement had been absent (see point $\left.\mathrm{E}\right)$. Since the choice of time periods and cohorts in the above discussion is arbitrary, the same relation holds more generally: births of order $j+1$ that occur at age $a$ at time $t$ to women who are parity $j$ in the reference year would have occurred at an age $\alpha=a-R_{j}^{T}(a, t)$ at 
time $\tau=t-R_{j}^{T}(a, t)$ in the absence of tempo changes after the reference year, where $R_{j}^{T}(a, t)$ reflects the cumulated tempo changes that have occurred between the reference year $T$ and time $t$.

The graphs in Figure 3 also reveal the importance of defining the postponement of fertility in the absence of changes in the level effect $q_{j}(t)$. In the case when $q_{j}(t)$ is held constant over time, the number of births occurring during the person years indicated by $\mathrm{ABCD}$ and AEFD are equal: a postponement of fertility at parity $j$ implies that births at parity $j$ are delayed but not foregone. This property is the essence of our definition of fertility postponement in Eq. (5). In periods when the level effect $q_{j}(t)$ is not constant, however, this equality between the number of births in the presence and absence of a postponement may not hold. In particular, if a delay of childbearing is combined with a declining level effect, the number of births during the person-years $\mathrm{ABCD}$ are less than the number during the person-years AEFD. This difference is due to the fact that the postponement of fertility leads to a 'stretching' of the person-years AEFD into the larger parallelogram $\mathrm{ABCD}$. This stretching implies that some exposure to births of order $j+1$ is moved to later time periods. During these later time periods, indicated by the area EBCF in Figure 3, the level effect $q_{j}(t)$ is lower than in earlier periods indicated by $\mathrm{ABCD}$. The declining level effect therefore reduces childbearing intensities in addition to the tempo-distortions caused by the delay of childbearing. The number of births occurring during the person-years $\mathrm{AEFD}$ and $\mathrm{ABCD}$, therefore, are no longer equal. This implies, for instance, that differences across cohorts in their mean age at first (or second) birth do not necessarily identify the extent of postponement that is relevant for the adjustment of childbearing intensities. In particular, these cohort differences can be due to either trends in the level effect or transformations of the age-pattern of childbearing intensities. Without further assumptions, pure cohort comparisons cannot separate these two aspects. The distinction, however, is essential since only the second aspect, changes in the agepattern of childbearing intensities, leads to tempo distortions.

On the basis of the above theoretical framework, we can now obtain our first and central result. This result specifies the formal relation between the intensity schedules $\phi_{j}^{T}(a)$ and $h_{j}(a, t)$ that reflect respectively the age-pattern of childbearing intensities in the presence and absence of a fertility postponement. The detailed proof of this result (and also of all subsequent results) is given in the Appendix. This proof relies on a change of variable in the integration of the probabilities $\pi_{j, T}^{h}\left(x, y^{\prime}\right)$ and $\pi_{j, T}^{\phi}\left(x, y^{\prime \prime}\right)$. This change transforms the variable of integration from the age $a$ and time $t$, indicating when births occur in the presence of a postponement, to the age $a-R_{j}^{T}(a, t)$ and time $t-R_{j}^{T}(a, t)$ that indicate when these births would have occurred in the absence of this postponement. The Jacobian of this transformation, which needs to be considered during this change of variable, equals $\left(1-\Delta_{a, t} R_{j}^{T}(a, t)\right)$. 
Result 1 Observed age-pattern of childbearing intensities: If births are postponed according to Definition 1, the observed age-pattern of childbearing intensities at parity $j$ are given by

$$
h_{j}(a, t)=\left(1-\Delta_{a, t} R_{j}^{T}(a, t)\right) \cdot \phi_{j}^{T}\left(a-R_{j}^{T}(a, t)\right) .
$$

Inserting this relation in Eq. (2) then yields the observed childbearing intensities at parity $j, m_{j}(a, t)$, as

$$
m_{j}(a, t)=q_{j}(t) \cdot\left(1-\Delta_{a, t} R_{j}^{T}(a, t)\right) \cdot \phi_{j}^{T}\left(a-R_{j}^{T}(a, t)\right) .
$$

The observed parity- $j$ childbearing intensities at age $a$ and time $t$ therefore consist of the period-specific level effect $q_{j}(t)$, the Jacobian of the transformation from $a$ and $t$ to $\alpha$ and $\tau$, and finally the standardized schedule $\phi_{j}^{T}\left(a-R_{j}^{T}(a, t)\right)$ evaluated at the age when births would have occurred in the absence of a fertility postponement after the reference year $T$.

Unfortunately, however, the decomposition of $m_{j}(a, t)$ in Result 1 does not yet have an interpretation in terms of 'adjusted childbearing intensities' and 'tempo distortions' caused by a postponement of fertility. This limitation is overcome in our next step:

Definition 2 Adjusted age-specific childbearing intensities: Denote as $m_{j}^{\prime}(a, t)$ the adjusted age-specific childbearing intensities at parity $j$ that are defined as

$$
m_{j}^{\prime}(a, t)=q_{j}(t) \cdot\left(1-\Delta_{a} R_{j}^{T}(a, t)\right) \cdot \phi_{j}^{T}\left(a-R_{j}^{T}(a, t)\right) .
$$

This definition, in combination with Assumption 4, implies that the adjusted childbearing intensities in reference period $T$ are equal to $m_{j}^{\prime}(a, T)=q_{j}(T) \cdot \phi_{j}^{T}(a)$.

The adjusted childbearing intensities in Eq. (8) are the product of three terms: the periodspecific level effect $q_{j}(t)$, a correction term $\left(1-\Delta_{a} R_{j}^{T}(a, t)\right)$, and the fertility rate $\phi_{j}^{T}(\alpha)$ that would have been observed at age $\alpha=a-R(a, t)$ at time $t$ if there had been no fertility postponement and no level effects. The correction term is necessary because age-period interactions in the postponement of fertility transform the shape of the intensity schedule. The term $\left(1-\Delta_{a} R_{j}^{T}(a, t)\right)$ entails that these transformations in the shape of the schedule in itself do not change the level of the integrated childbearing intensities $\int m_{j}^{\prime}(a, t) d a$. All changes in this level are attributed to the period-specific level effects $q_{j}(t)$ :

Result 2 Level of fertility: The adjusted childbearing intensities reflect the level effect $q_{j}(t)$ since

$$
\int m_{j}^{\prime}(a, t) d a=q_{j}(t) .
$$


In the presence of a fertility postponement, however, the observed childbearing intensities are distorted and they do not reflect the level of fertility at parity $j$ and time $t$. In particular, the observed childbearing intensities at parity $j$ in the presence of a fertility postponement are only a fraction of the intensities that would be observed at time $t$ in the absence of tempo changes. The observed intensities can thus substantially underestimate the level of fertility at parity $j$ in periods with postponed childbearing:

Result 3 Observed versus adjusted childbearing intensities: The observed childbearing intensities $m_{j}(a, t)$ and the adjusted childbearing intensities $m_{j}^{\prime}(a, t)$ are related as

$$
m_{j}(a, t)=\left(1-r_{j}(a, t)\right) \cdot m_{j}^{\prime}(a, t),
$$

where the age-and-period specific tempo change $r_{j}(a, t)$ is defined as

$$
r_{j}(a, t)=\frac{\Delta_{t} R_{j}^{T}(a, t)}{1-\Delta_{a} R_{j}^{T}(a, t)} .
$$

Finally, we point out that the above definition of adjusted childbearing intensities $m^{\prime}(a, t)$, as well as the relations in Results 2-3, are independent of the choice of the reference year $T$. The above model can therefore be restated in terms of any other arbitrary year $T^{\prime}$ :

Result 4 Choice of reference year: The choice of a reference year $T$ can be altered to any other reference year $T^{\prime}$ by specifying the standardized schedule $\phi_{j}^{T^{\prime}}(a)$ as $\phi_{j}^{T^{\prime}}(a)=$ $\left(1-\left.\Delta_{a} R_{j}^{T}(a, t)\right|_{t=T^{\prime}}\right) \cdot \phi_{j}^{T}\left(a-R_{j}^{T}\left(a, T^{\prime}\right)\right)$ and redefining the cumulated tempo change $R_{j}^{T^{\prime}}(a, t)$ so that it satisfies $R_{j}^{T}(a, t)=R_{j}^{T^{\prime}}(a, t)+R_{j}^{T}\left(a-R^{T^{\prime}}(a, t), T^{\prime}\right)$. After this redefinition of the reference year, all changes in the age-pattern of fertility are then expressed relative to the schedule $\phi_{j}^{T^{\prime}}(a)$, and this schedule is proportional to the adjusted childbearing intensities in the new reference year $T^{\prime}$.

\subsection{Measuring and adjusting for tempo changes}

Our previous discussion has been very general and allowed for virtually unrestricted postponement patterns and cumulated tempo changes. The empirical implementation of the above framework, however, requires specific assumptions about the functional form of the cumulated tempo $R_{j}^{T}(a, t)$. Without such specific assumptions, the standardized schedule $\phi_{j}^{T}(a)$ and the cumulated and age-and-period specific tempo changes, $R_{j}^{T}(a, t)$ and $r_{j}(a, t)$ are not empirically identified.

In this paper we adopt the specification in Kohler and Philipov (2001) that allows for both mean changes $\gamma_{j}(t)$, leading to an increase in the mean age of the parity- $j$ intensity schedule, and variance changes $\delta_{j}(t)$, leading to an increase of the variance of the intensity schedule (see KP for a more detailed discussion of this framework): 
Assumption 5 Fertility postponement in terms of mean and variance changes: The age-and-period specific tempo changes $r_{j}(a, t)$ are given by

$$
r_{j}(a, t)=\gamma_{j}(t)+\delta_{j}(t)\left[a-\left(\bar{a}_{j, T}+G_{j}^{T}(t)\right)\right],
$$

where $\gamma_{j}(t)$ and $\delta_{j}(t)$ are smooth functions of time, and $G_{j}^{T}(t)=\int_{T}^{t} \gamma_{j}(z) d z$. We assume that $r_{j}(a, t)<1$ at all ages a at which the childbearing intensities are positive.

The cumulated tempo changes, $R_{j}^{T}(a, t)$, that correspond to age-and-period specific tempo change $r_{j}(a, t)$ in Eq. (11), is specified by

$$
R_{j}^{T}(a, t)=G_{j}^{T}(t)+\left[a-\left(\bar{a}_{j, T}+G_{j}^{T}(t)\right)\right] \cdot\left(1-e^{-D_{j}^{T}(t)}\right),
$$

where $D_{j}^{T}(t)=\int_{T}^{t} \delta_{j}(z) d z$.

The reason that motivates the above choice for the age-and-period specific tempo change $r_{j}(a, t)$ and the cumulated tempo $R_{j}^{T}(a, t)$ is apparent from the following result about the development of the mean age and variance of the adjusted intensity schedule over time:

Result 5 Mean age and variance of adjusted intensity schedule: The mean age $\bar{a}_{j}(t)$ and the variance $s_{j}^{2}(t)$ of the adjusted intensity schedule $m_{j}^{\prime}(a, t)$ are given by

$$
\begin{aligned}
\bar{a}_{j}(t) & =\int a \cdot m_{j}^{\prime}(a, t) d a / q_{j}(t)=\bar{a}_{j, T}+G_{j}^{T}(t) \\
d \bar{a}_{j}(t) / d t & =\gamma_{j}(t) \\
s_{j}^{2}(t) & =\int\left(a-\bar{a}_{j}(t)\right)^{2} \cdot m_{j}^{\prime}(a, t) d a / q_{j}(t)=s_{j, T}^{2} \cdot e^{2 \cdot D_{j}^{T}(t)} \\
d \log s_{j}^{2}(t) / d t & =2 \delta_{j}(t),
\end{aligned}
$$

where $\bar{a}_{j, T}$ is the mean age and $s_{j, T}^{2}$ is the variance of the adjusted intensity schedule $m_{j}^{\prime}(a, T)$ at time $T$. Moreover, inserting the first expression for $\bar{a}_{j}(t)$ into the specification of $r_{j}(a, t)$ in Eq. (11) yields a simplified version of the age-and period specific tempo change as

$$
r_{j}(a, t)=\gamma_{j}(t)+\delta_{j}(t)\left[a-\bar{a}_{j}(t)\right]
$$

The specification of the fertility postponement in Assumption 5 thus leads to particularly simple changes in the mean age and variance of the adjusted intensity schedule $m_{j}^{\prime}(a, t)$ over time. Specifically, the incremental increase in the mean age at time $t$ is given by $\gamma_{j}(t)$, and the incremental relative increase in the standard deviation is given by $\delta_{j}(t)$. Moreover, the term $G_{j}^{T}(t)$ represents the total increase in the mean age of the intensity 
schedule between the reference year $T$ and some later time $t$, and the term $\exp \left[D_{j}^{T}(t)\right]$ measures the corresponding relative increase in the standard deviation.

The central insight provided by Result 5 is that the relevant information for the assessment of tempo distortions is provided by the moments of the adjusted parity-specific intensity schedules (a similar approach to model tempo changes is also used in Keilman 1994). The above specification in terms of mean changes $\gamma_{j}(t)$ and variance changes $\delta_{j}(t)$ is therefore particularly convenient for the empirical estimation of tempo changes. Moreover, the analog of the BF framework, which implies only shifts in the mean age of the fertility schedules but no changes in the variances, emerges within the above specification when $\delta_{j}(t)$ is equal to zero at all times.

\section{Parity Progression Measures under Alternative Post- ponement Scenarios}

In this section we utilize tempo-adjusted childbearing intensities defined in Section 3 for two main applications to (a) provide improved synthetic-cohort period measures of fertility and $(b)$ to complete the fertility of cohorts, who are still in childbearing ages, under alternative postponement scenarios that determine future patterns of tempo changes. We begin our formal development with application to cohort completion, and we then present the measurement of period fertility as a special case of cohort completion that considers cohorts at the beginning of childbearing ages under the postponement stops scenario.

\subsection{Extrapolation of fertility postponement to future periods}

The primary task in the analyses of cohort fertility under different postponement scenarios is to establish a relation between the childbearing intensities experienced by the cohorts and the adjusted intensity schedule in a reference year. Since the adjusted intensity schedule is a product of a level effect and an age-pattern of childbearing intensities, these two determinants - combined with specific assumptions about future tempo changes-can be used to characterize the intensities that are experienced by cohorts. We can therefore provide general formulas for cohort completion conditional on the parity-specific level effect in the reference year and an arbitrary postponement scenario. In the present paper, we focus on two specific scenarios that are particularly pertinent. The postponement stops scenario assumes that any postponement that occurs in the reference year $T$ comes to a halt and that there is no further postponement of fertility during the life-course of the cohort. In contrast, the postponement continues scenario assumes that the mean- and 
variance changes observed in the reference year $T$ prevail in the future. The period intensity schedule therefore continues to be shifted to later ages during the life-course of the cohorts, and the annual extent of the shift equals the mean and variance change that is observed in the reference year $T$. Both scenarios assume that the parity-specific level effects in the reference year, $q_{j}(T)$, continue to prevail in the future for all $t \geq T$.

We illustrate these two different postponement scenarios in the Lexis diagrams in Figure 4. In this illustration we choose the year 2000 as the reference year for the calculations. Childbearing intensities are observed prior to this year, and the intensity schedule for the year 2000 is the last available period information. We indicate these childbearing intensities and their age-pattern in a simplified manner through an age range that indicates the minimum, mean and maximum of the intensity schedule. Moreover, the example in Figure 4 assumes that there is a delay of fertility during the years 1980-2000. This postponement is reflected through the gradual upward-shift of the age-range of childbearing intensities (for simplicity we assume that there is only a mean change and no variance change). This delay prior to and at $T=2000$ implies that the observed intensities in the reference year are subject to tempo distortions. These distortions can be eliminated by the adjustment discussed in the previous section. The resulting adjusted intensity schedule for the reference year $T$ is then the basis for specifying the childbearing intensities that are experienced by the synthetic cohort.

If the postponement stops in the reference year $T$, then there are no further changes in the age-pattern of childbearing intensities during the periods $t \geq T$ (Figure 4a). Thus, there are no tempo-distortions at time $t \geq T$, and the period intensity schedules at time $t \geq T$ are equal to the adjusted intensity schedule in the reference year. Consider now the cohort born in 1985 who is age 15 in the year 2000. If the postponement stops scenario prevails, this cohort experiences the childbearing intensities along the diagonal cohort line AB. These childbearing intensities, however, are identical to the adjusted intensities observed in the reference year $T$. The analysis along the line $\mathrm{AB}$ is therefore equivalent to the analysis of the adjusted intensity schedule at time $T$.

It is important to observe, however, that this postponement stops scenario represents a break with the earlier development of birth timing that has been characterized by a delay in childbearing. This discontinuity in the evolution of childbearing intensities is avoided in the postponement continues scenario (Figure 4b). In this scenario, the mean change (and also the variance change) observed in the reference year $T$ continues to prevail during the period $t \geq T$. The age-range of childbearing intensities therefore continues to be shifted upward, and the pace of this future postponement follows a constant annual rate that equals the mean change in the reference year $T=2000$. In order to obtain the fertility pattern of a cohort in this postponement continues scenario, we also need to integrate the childbearing intensities along the cohort line in the Lexis diagram. The integration along this line, however, needs to account for the shifting age-pattern of childbearing intensities 
Demographic Research - Volume 6, Article 6

Figure 4: Postponement stops and postponement continues scenario in the Lexis diagram

(a) postponement stops

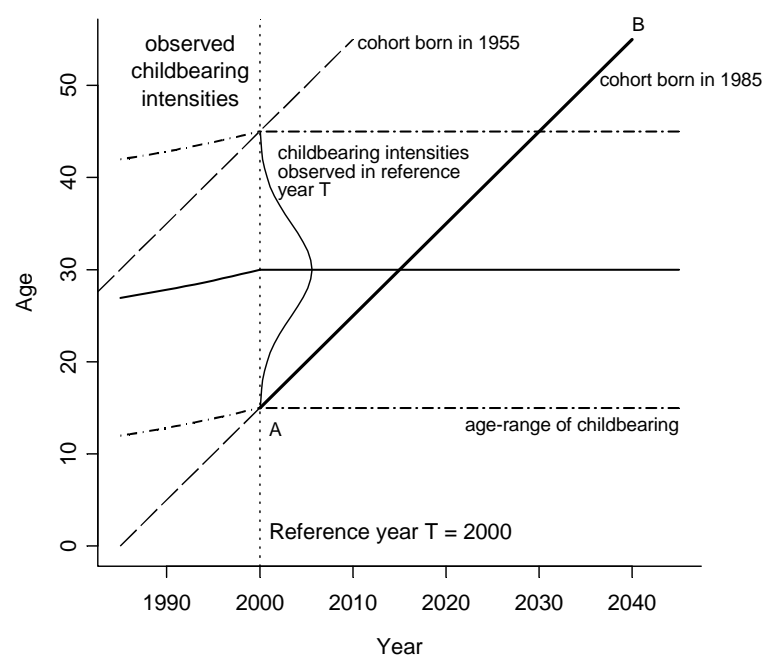

(b) postponement continues

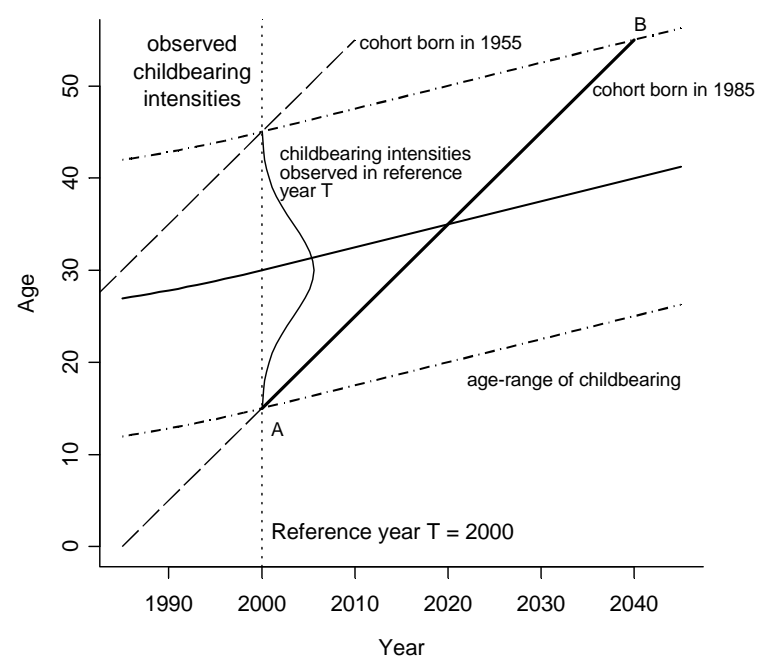


after the reference year. The fertility of a cohort that is age 15 in the year $T=2000$, for instance, is obtained by tracing the childbearing intensities along the line AB in Figure 4(b). In contrast to the postponement stops scenario, however, the intensities along the line $\mathrm{AB}$ are no longer equal to the adjusted childbearing intensities in the reference year. If postponement continues, the mean age of the period intensity schedule is continuously shifted upwards, and the childbearing intensities that are experienced by the cohort along the line $\mathrm{AB}$ are a transformation of the adjusted intensity schedule observed in the year $T=2000$.

Fortunately, we can capture this transformation by extending the formal framework for the postponement of fertility that we have developed in the previous sections. In particular, we can use the observed tempo and variance changes in the reference year $T$ to specify the cumulated tempo changes for all periods $t \geq T$. The fertility measures for a cohort therefore follow from the observed childbearing intensities in three conceptually distinct steps: (a) the measurement of and adjustment for tempo-distortions in the observed childbearing intensities; $(b)$ the projection of all childbearing intensities for $t \geq T$ based on the adjusted intensity schedule in the reference year and assumptions about the future postponement of fertility; $(c)$ the calculation of completed cohort fertility by integrating the intensities obtained in $(b)$ along cohort lines in the Lexis diagram (e.g., along the line $\mathrm{AB}$ in Figure 4).

In the following we formalize the above discussion of Figure 4, and we establish the childbearing intensities that would be observed after the reference year $T$ if the level of the reference year and some arbitrary tempo change and variance change prevails for all times thereafter. These childbearing intensities follow directly from our earlier Result 1 and Definition 2:

Result 6 Extrapolation of fertility postponement to future periods: Denote as $m_{j}^{s}(a, t)$ the parity- $j$ childbearing intensities that would be observed at age a and time $t \geq T$ if (a) the level effect $q_{j}(T)$ observed in the reference year $T$ prevails for all $t \geq T$, and (b) fertility at parity $j$ continues to be postponed with a constant annual mean change $\gamma_{j}^{s}$ and variance change $\delta_{j}^{s}$ for all $t \geq T$. In addition, denote as $R_{j}^{s}(a, t)$ the cumulated tempo and as $r_{j}^{s}(a, t)$ the age-and-period specific tempo change that are obtained from Eqs. (11-12) using $\gamma_{j}(t)=\gamma_{j}^{s}$ and $\delta_{j}(t)=\delta_{j}^{s}$ for all $t \geq T$. Then

$$
\begin{aligned}
m_{j}^{s}(a, t) & =q_{j}(T) \cdot\left(1-\Delta_{a, t} R_{j}^{s}(a, t)\right) \cdot \phi_{j}^{T}\left(a-R_{j}^{s}(a, t)\right) \\
& =\left(1-\Delta_{a, t} R_{j}^{s}(a, t)\right) \cdot m_{j}^{\prime}\left(a-R_{j}^{s}(a, t), T\right) .
\end{aligned}
$$

The corresponding adjusted intensity schedule $m_{j}^{s \prime}(a, t)$ for all $t \geq T$ is given by

$$
\begin{aligned}
m_{j}^{s \prime}(a, t) & =\left(1-r_{j}^{s}(a, t)\right)^{-1} \cdot m_{j}^{s}(a, t) \\
& =q_{j}(T) \cdot\left(1-\Delta_{a} R_{j}^{s}(a, t)\right) \cdot \phi_{j}^{T}\left(a-R_{j}^{s}(a, t)\right) .
\end{aligned}
$$


The mean age $\bar{a}_{j}^{s}(t)$ and variance $s_{j, s}^{2}(t)$ of the adjusted fertility schedules $m_{j}^{s \prime}(a, t)$ at time $t$ are given by $\bar{a}_{j}^{s}(t)=\bar{a}_{j, T}+\gamma_{j}^{s} \cdot(t-T)$ and $s_{j, s}^{2}(t)=s_{j, T}^{2} e^{2 \cdot \delta_{j}^{s} \cdot(t-T)}$, where $\bar{a}_{j, T}$ and $s_{j, T}^{2}$ are the mean age and variance of the adjusted intensity schedule $m_{j}^{\prime}(a, T)$ in the reference year T. Moreover, the integrals of the observed and adjusted childbearing intensities for all $t \geq T$ are constant over time and equal respectively $\int m_{j}^{s}(a, t) d a=$ $\left(1-\gamma_{j}^{s}\right) \cdot q_{j}(T)$ and $\int m_{j}^{s \prime}(a, t) d a=q_{j}(T)$.

The above result therefore allows us to calculate hypothetical intensity schedules that would be observed at some time $t \geq T$ if $(a)$ the level effect observed in the reference year $T$ at parity $j$ prevails in the future, and if $(b)$ fertility is subject to a postponement pattern with a constant annual mean change $\gamma_{j}^{s}$ and variance change $\delta_{j}^{s}$ for all $t \geq T$. The mean age of the adjusted intensity schedule at parity $j$ then increases annually by $\gamma_{j}^{s}$ years, and the standard deviation grows exponentially at an annual rate of $\delta_{j}^{s}$. [Note 7] Moreover, the integral of the adjusted childbearing intensities remains constant for all $t \geq T$ and equals the level effect $q_{j}(T)$ in the reference year $T$.

We subsequently use the phrase that calculations pertain to the 'period- $T$ cohort of age $x$ ' in order to describe the following assumption about the fertility experience of the cohort for which parity progression measures are calculated:

Assumption 6 Parity progression measures for the period-T cohort of age $x$ : In our calculations of parity progression measures we assume a cohort, denoted period-T cohort of age $x$, who $(a)$ is age $x$ in the reference year $T$ and $(b)$ experiences the childbearing intensities that are obtained by extrapolating the level of fertility and the pace of fertility postponement observed in the reference year $T$ to all times $t \geq T$. That is, we assume that women in the period-T cohort of age $x$ are subject to the childbearing intensities $m_{j}^{s}(a, t)$ defined in Eq. (14) when they attain age a at time $t=T+(a-x)$.

We use the term 'period-T cohort of age $x$ and parity $j$ ' if we additionally condition on parity and consider women who are at exact parity $j$ in the reference year $T$.

The maximum feasible age at childbearing ('biological limit') is given by $\omega$. We assume that this age does not imply a restriction for the postponement of fertility in our analyses, i.e., we assume that the childbearing intensities defined in Eq. (14) satisfy $m_{j}^{s}(a, T+a-x)=0$ for all $a \geq \omega$. [Note 8]

Our two basic approaches to determine the pace of fertility postponement in the synthetic cohort are specified as follows: (a) 'extrapolate' the postponement pattern observed at time $T$, i.e., assume that the mean change $\gamma_{j}^{s}$ and variance change $\delta_{j}^{s}$ during the life-course of the cohort equal the values of $\gamma_{j}(T)$ and $\delta_{j}(T)$ observed in the reference year $T$ for all parities $j ;(b)$ assume that there are no further postponements of fertility subsequent to time $T$, i.e., assume that $\gamma_{j}^{s}$ and $\delta_{j}^{s}$ are zero for all parities $j$. The former represents the 
postponement continues and the latter the postponement stops scenario. [Note 9] In our subsequent notation, we indicate the postponement stops scenario as ' $\gamma_{j}^{s}=0, \delta_{j}^{s}=0$ ' and the postponement continues scenario as ' $\gamma_{j}^{s}=\gamma_{j}(T), \delta_{j}^{s}=\delta_{j}(T)$ '. For simplicity, we often drop the subscript $j$ for fertility measures that combine several birth parities, and we use ' $\gamma^{s}, \delta^{s}$ ' as a shorthand notation for ' $\gamma_{0}^{s}, \gamma_{1}^{s}, \ldots, \delta_{0}^{s}, \delta_{2}^{s}, \ldots$ '.

\subsection{Conditional parity progression probabilities}

The primary building block for deriving the parity progression ratios for the period- $T$ cohort of age $x$ is the conditional parity progression probability that is calculated conditionally on a postponement scenario described by $\gamma_{j}^{s}$ and $\delta_{j}^{s}$ :

Result 7 Conditional parity progression probability: Consider a woman in the period- $T$ cohort of age $x$ and parity $j$. This woman is exposed to a birth of order $j+1$ from age $x$ and time $T$ onwards. Denote as $p_{j}^{T}\left(x, y \mid \gamma^{s}, \delta^{s}\right)$ the probability that this woman attains a parity of at least $j+1$ at age $y$ (with $y \geq x$ ) by having at least one additional child prior to age $y$.

Integrating the childbearing intensities $m_{j}^{s}(a, t)$ experienced by the period-T cohort of age $x$ (see Eq. 14) yields

$$
\begin{aligned}
p_{j}^{T}\left(x, y \mid \gamma_{j}^{s}, \delta_{j}^{s}\right) & =1-\exp \left[-\int_{x}^{y} m_{j}^{s}(a, T+a-x) d a\right] \\
& =1-\exp \left[-\int_{x}^{y-R_{j}^{s}(y, T+(y-x))} m_{j}^{\prime}(a, T) d a\right] .
\end{aligned}
$$

In the specific case $y=\omega$, the conditional lifetime parity progression probability $p_{j}^{T}(x, \omega \mid$ $\gamma_{j}^{s}, \delta_{j}^{s}$ ) reflects the probability that a woman in the period-T cohort of age $x$ and parity $j$ has at least one additional child and progresses to parity $j+1$ or higher. This probability is given by the integral of the adjusted parity-j age-specific childbearing intensities at time $T$ from age $x$ onwards,

$$
p_{j}^{T}\left(x, \omega \mid \gamma_{j}^{s}, \delta_{j}^{s}\right)=1-\exp \left[-\int_{x}^{\infty} m_{j}^{\prime}(a, T) d a\right],
$$

and is thus independent of the assumption about $\gamma_{j}^{s}$ and $\delta_{j}^{s}$.

An important insight from Eq. (17) is that the conditional parity progression probabilityindependent of the assumption about the future postponement of fertility — can be calculated directly from the adjusted childbearing intensities in the reference year $T$. Different 
assumptions about future mean- and variance changes at parity $j$ affect only the upper limit of the integral in Eq. (17). The conditional parity progression probability-as well as all subsequent parity progression measures - can therefore be obtained without ever calculating the childbearing intensities $m_{j}^{s}(a, t)$ (see Eq. 14) that are experienced by the period- $T$ cohort of age $x$. This feature makes the calculation of different postponement scenarios particularly simple. All postponement scenarios are obtained by integrating the adjusted intensity schedule in the reference year $T$, and the assumptions about the future postponement of fertility only need to be considered in the upper limit of the integral.

The above result has several interesting implications. First, the exponent of the integral of the adjusted childbearing intensities yields a proper assessment of conditional parity progression probabilities in cohorts. Second, in order to estimate $p_{j}^{T}\left(x, \omega \mid \gamma_{j}^{s}, \delta_{j}^{s}\right)$, i.e., the probability that a woman who is age $x$ and parity $j$ at time $T$ has another child, it is sufficient to know the adjusted age-specific childbearing intensities $m_{j}^{\prime}(a, T)$. No further assumptions about the future path of fertility postponement are necessary. Third, since in periods of fertility postponement $m_{j}^{\prime}(a, t)$ exceeds the observed intensities $m_{j}(a, t)$, a calculation of the conditional parity progression probability $p_{j}^{T}\left(x, y \mid \gamma_{j}^{s}, \delta_{j}^{s}\right)$ on the basis of the observed childbearing intensities would underestimate the probability to have another child:

Result 8 Distortion of conditional parity progression probability due to tempo changes: The calculation of the conditional parity progression probability $p_{j}^{T}\left(x, \omega \mid \gamma_{j}^{s}, \delta_{j}^{s}\right)$ in Eq. (17) based on the observed instead of the adjusted childbearing intensities is distorted. In the specific case when $\delta_{j}(T)=0$ and $\delta_{j}^{s}=0$, i.e., in the absence of variance changes for parity $j$, the calculation based the observed intensities yields 1 - (1$\left.p_{j}^{T}\left(x, y \mid \gamma_{j}^{s}, \delta_{j}^{s}\right)\right)^{1-\gamma_{j}(T)}$. This probability is smaller than the correct value $p_{j}^{T}(x, y \mid$ $\left.\gamma_{j}^{s}, \delta_{j}^{s}\right)$ obtained from the adjusted childbearing intensities whenever the mean change $\gamma_{j}(T)$ at time $T$ is positive. Moreover, the calculations based on the observed intensities always underestimates the correct value of the lifetime birth probability, $p_{j}^{T}\left(x, \omega \mid \gamma_{j}^{s}, \delta_{j}^{s}\right)$, whenever $\gamma_{j}(T)>0$ and $\delta_{j}(T) \geq 0$.

The failure to account for tempo distortions therefore leads to erroneous inferences of period parity progression probabilities and related measures.

\subsection{Conditional parity progression ratios}

In this section we derive parity progression ratios and some related measures from the conditional parity progression probabilities introduced above. These calculations of parity progression ratios follow mostly from the application of standard multi-state population theory (e.g., Hoem and Jensen 1982; Schoen 1988). [Note 10] We begin with the parity distribution attained by the period- $T$ cohort of age $x$ : 
Result 9 Conditional Parity distribution in a cohort: Consider a woman in the period$T$ cohort of age $x$ and parity $j$. Denote as the parity distribution ${ }_{n} D_{j}^{T}\left(x, y \mid \gamma^{s}, \delta^{s}\right)$ the probability that this woman is at exact parity $j+n$ at age $y$ (with $n \geq 0$ and $y \geq x$ ) conditional on the postponement scenario given by $\gamma_{j}^{s}, \gamma_{j+1}^{s}, \ldots \gamma_{j+n}^{s}$ and $\delta_{j}^{s}, \delta_{j+1}^{s}, \ldots \delta_{j+n}^{s}$. For simplicity, we denote this postponement scenario in our notation as ' $\gamma^{s}, \delta^{s}$.'

The probability of remaining at parity $j$ until at least age $y$ then follows by setting $n=0$ and is given by ${ }_{0} D_{j}^{T}\left(x, y \mid \gamma^{s}, \delta^{s}\right)=\exp \left[-\int_{x}^{y} m_{j}^{s}(a, T+a-x) d a\right]=1-$ $p_{j}^{T}\left(x, y \mid \gamma_{j}^{s}, \delta_{j}^{s}\right)$. The probability of attaining an exact parity $j+n$ with $n>0$ is obtained via

$$
\begin{aligned}
{ }_{n} D_{j}^{T}\left(x, y \mid \gamma^{s}, \delta^{s}\right)= & \int_{x}^{y}{ }_{n-1} D_{j}^{T}\left(x, a \mid \gamma^{s}, \delta^{s}\right) \cdot m_{j+n-1}^{s}(a, T+a-x) . \\
& s_{j+n}^{T}\left(a, y \mid \gamma_{j+n}^{s}, \delta_{j+n}^{s}\right) d a,
\end{aligned}
$$

where $s_{j+n}^{T}\left(a, y \mid \gamma_{j+n}^{s}, \delta_{j+n}^{s}\right)$ is the probability of remaining at parity $j+n$ until age $y$, conditional on being at exact parity $j+n$ at age $a$; this probability is calculated as

$$
\begin{aligned}
s_{j+n}^{T}\left(a, y \mid \gamma_{j+n}^{s}, \delta_{j+n}^{s}\right) & =\exp \left[-\int_{a}^{y} m_{j+n}^{s}(a, T+a-x) d a\right] \\
& =\frac{1-p_{j+n}^{T}\left(x, y \mid \gamma_{j+n}^{s}, \delta_{j+n}^{s}\right)}{1-p_{j+n}^{T}\left(x, a \mid \gamma_{j+n}^{s}, \delta_{j+n}^{s}\right)} .
\end{aligned}
$$

Consider, for example, the parity distribution of the cohort of women who are age 15 and parity 0 at time $T$. In terms of the above notation this implies $x=15$ and $j=0$. The fraction of these women who are still childless at age $y$ is then given by ${ }_{0} D_{0}^{T}\left(15, y \mid \gamma^{s}, \delta^{s}\right)$ and follows directly from the probability of 'surviving' in the childless state. For first and higher order births the parity distribution ${ }_{n} D_{0}^{T}\left(15, y \mid \gamma^{s}, \delta^{s}\right)$ needs to be solved iteratively. For instance, for first birth (i.e., $n=1$ ), the terms within the integral in Eq. (18) represent respectively the fraction of the initial cohort who is at exact parity 0 at age $a$, the risk of experiencing the first birth at time $T+a-x$ when the cohort is age $a$ (see Eq. 14), and the probability of not having another child prior to reaching age $y$. The advantage of the above calculation is that all terms entering Eq. (18) can be obtained from the conditional parity progression probabilities, which in turn are calculated by simply integrating the adjusted intensity schedules in the reference year $T$ (independent of the assumptions about the future postponement of fertility). [Note 11]

The final parity distribution conditional on the postponement scenario follows from the above calculations as:

Result 10 Final parity distribution in the cohort: Consider the period-T synthetic cohort of age $x$ and parity $j$. The final parity distribution in this cohort is given by ${ }_{n} D_{j}^{T}(x, \omega \mid$ $\left.\gamma^{s}, \delta^{s}\right)$, where $\omega$ is the highest feasible age at child-birth. 
Closely related to the parity distribution in Results $9-10$ is the period lifetime birth probability of Park $(1967,1976)$, which is extended here to the case of a continued postponement of fertility:

Result 11 Conditional period birth probability of $\boldsymbol{n}$ additional children: Consider the period-T cohort of age $x$ and parity $j$. Denote as ${ }_{n} F_{j}^{T}\left(x, y \mid \gamma^{s}, \delta^{s}\right)$ the period birth probability of $n$ additional children that represents the probability that a woman in this cohort gives birth to at least $n$ additional children-and thus reaches parity $j+n$ or higher-prior to age $y$ (with $y \geq x$ ), conditional on the postponement scenario given by $\gamma^{s}$ and $\delta^{s}$.

For $n=0$ we define ${ }_{0} F_{j}^{T}\left(x, y \mid \gamma^{s}, \delta^{s}\right)=1$ since a woman who is at parity $j$ at age $x$ always has $j$ or more children when she reaches age $y$. For $n>0$ we obtain

$$
{ }_{n} F_{j}^{T}\left(x, y \mid \gamma^{s}, \delta^{s}\right)=\int_{x}^{y}{ }_{n-1} D_{j}^{T}\left(x, a \mid \gamma^{s}, \delta^{s}\right) \cdot m_{j+n-1}^{s}(a, T+a-x) d a .
$$

Moreover, we denote as the period lifetime birth probability of $\boldsymbol{n}$ additional children the special case ${ }_{n} F_{j}^{T}\left(x, \omega \mid \gamma^{s}, \delta^{s}\right)$ that is obtained by setting the second age limit to the maximum feasible age at childbearing $\omega$.

The period birth probabilities and parity distributions are related as

$$
{ }_{n} F_{j}^{T}\left(x, y \mid \gamma^{s}, \delta^{s}\right)=\sum_{k \geq n}{ }_{k} D_{j}^{T}\left(x, y \mid \gamma^{s}, \delta^{s}\right)
$$

Finally, we can use the above framework to derive parity progression ratios that are free of tempo-distortions and allow for a continued postponement of childbearing. Moreover, we can use these parity progression ratios to calculate the completed cohort fertility:

Result 12 Conditional parity progression ratios and conditional total fertility: Consider again the period-T cohort of age $x$ and parity $j$. Denote as ${ }_{n} \pi_{j}^{T}\left(x \mid \gamma^{s}, \delta^{s}\right)$ the conditional parity progression ratio that equals the probability that a woman in this cohort gives birth to at least $n$ additional children, conditional on giving birth to at least $n-1$ additional children and conditional on the postponement scenario given by $\gamma^{s}$ and $\delta_{s}$.

If $n=1$, the parity progression ratio follows directly from the lifetime birth probability as ${ }_{1} \pi_{j}^{T}\left(x \mid \gamma^{s}, \delta^{s}\right)={ }_{1} F_{j}^{T}\left(x, \omega \mid \gamma^{s}, \delta^{s}\right)$. For higher parities $n>1$, the period parity progression ratios follow as

$$
{ }_{n} \pi_{j}^{T}\left(x \mid \gamma^{s}, \delta^{s}\right)=\frac{{ }_{n} F_{j}^{T}\left(x, \omega \mid \gamma^{s}, \delta^{s}\right)}{{ }_{n-1} F_{j}^{T}\left(x, \omega \mid \gamma^{s}, \delta^{s}\right)}
$$


Completed fertility for the period-T cohort of age $x$ and parity $j$ is given by the conditional index of total fertility, $T F_{j}^{T}\left(x \mid \gamma^{s}, \delta^{s}\right)$, that is calculated as

$$
\begin{aligned}
T F_{j}^{T}\left(x \mid \gamma^{s}, \delta^{s}\right) & =j+\sum_{k \geq 1}{ }_{k} F_{j}^{T}\left(x, \omega \mid \gamma^{s}, \delta^{s}\right) \\
& =j+{ }_{1} \pi_{j}^{T}\left(x \mid \gamma^{s}, \delta^{s}\right)+{ }_{1} \pi_{j}^{T}\left(x \mid \gamma^{s}, \delta^{s}\right) \cdot{ }_{2} \pi_{j}^{T}\left(x \mid \gamma^{s}, \delta^{s}\right)+\ldots
\end{aligned}
$$

\subsection{Conditional cohort mean age at birth}

Our analyses in the previous sections have emphasized various indicators of completed cohort fertility such as parity progression ratios, lifetime birth probabilities and the index of total fertility. The relevant indicators for changes in the timing of fertility in that context are provided by the intensity schedule mean age and variance at different parities (see Result 5). It is important to note, however, that the mean age and variance of the parity- $j$ intensity schedule are not equivalent to the average age at birth of order $j+1$ in either real or synthetic cohorts. Similarly, the mean change $\gamma_{j}$ does not represent the change in the mean age at birth in synthetic or real cohorts over time. The parity-specific mean age and variance of the intensity schedules are therefore merely characteristics of the age-pattern of the childbearing intensities at different parities. They provide the basis for detecting changes in the timing of fertility, but they do not have a cohort interpretation. The distribution of births of a given order in real or synthetic cohorts differs from the age-pattern of the childbearing intensities and is obtained using the translation formulae for non-repeatable events (Keilman 1994; Keilman and van Imhoff 1995). In order to obtain the mean age at birth for the completed cohorts, we therefore extend some existing translation results and apply them to the framework developed in this paper. Moreover, we are able to develop an explicit relation for the mean age at first birth as a function of the annual mean change $\gamma_{0}^{s}$ at parity zero.

We use the term conditional cohort mean age at birth $(C M A B)$ in order to distinguish the average age at birth from the intensity schedule mean age. The basic idea underlying the $C M A B$ was initially introduced by Hajnal $(1947,1953)$ and applied to first marriage. These results for first marriage can be directly transferred to first births, and they apply in an analog fashion to second and higher parity births. In this paper we extend this framework to allow for different postponement scenarios, including a continued delay of childbearing during a cohort's life-course.

Result 13 Conditional cohort mean age at birth of order $j$ : Consider the period-T synthetic cohort of age $x$ and parity $j$. Denote as the synthetic cohort mean age ${ }_{n} C M A B_{j}^{T}(x)$ $\left.\gamma^{s}, \delta^{s}\right)$ the average age at birth of order $j+n$ in this cohort as a function of the annual parity-specific mean changes $\gamma_{j+k}^{s}$ and variance changes $\delta_{j+k}^{s}$, where $k=0, \ldots, n-1$, 
that prevail during the life-course of this cohort. Then

$$
\begin{aligned}
{ }_{n} C M A B_{j}^{T}\left(x \mid \gamma^{s}, \delta^{s}\right)= & \frac{1}{{ }_{n} F_{j}^{T}\left(x, \omega \mid \gamma^{s}, \delta^{s}\right)} \cdot\left[\int_{x}^{\omega} a \cdot{ }_{n-1} D_{j}^{T}\left(x, a \mid \gamma^{s}, \delta^{s}\right) .\right. \\
& \left.m_{j+n-1}^{s}(a, T+a-x) d a\right] .
\end{aligned}
$$

For the special case when $n=1, E q$. (20) can be transformed into Hajnal's formula as

$$
\begin{aligned}
{ }_{1} C M A B_{j}^{T}\left(x \mid \gamma^{s}, \delta^{s}\right)= & x+\frac{1}{1-{ }_{0} D_{j}^{T}\left(x, \omega \mid \gamma^{s}, \delta^{s}\right)} \cdot\left[\int_{x}^{\omega}{ }_{0} D_{j}^{T}\left(x, a \mid \gamma^{s}, \delta^{s}\right) d a-\right. \\
& \left.(\omega-x) \cdot{ }_{0} D_{j}^{T}\left(x, \omega \mid \gamma^{s}, \delta^{s}\right)\right] .
\end{aligned}
$$

The above conditional cohort mean age at birth therefore provides a translation of the period fertility pattern into the corresponding cohort birth timing. For most applications, the above calculations will be performed for relatively young childless cohorts so that $x$ equals, say, 15 years and $j$ is set to zero. For our later projection of cohort fertility, however, the more general framework introduced above is useful.

The overall mean age at birth, which accounts for births of all orders, then follows by averaging across birth-orders as:

Result 14 Overall conditional cohort mean age at birth: Consider again the period-T cohort of age $x$ and parity $j$. The overall conditional mean age (including all births of order $j+1$ and higher) in this cohort is given by the weighted average

$$
\begin{aligned}
M A B_{j}^{T}\left(x \mid \gamma^{s}, \delta^{s}\right)= & \frac{1}{T F_{j}^{T}\left(x \mid \gamma^{s}, \delta^{s}\right)-j}\left[\sum_{n \geq 1}{ }_{n} F_{j}^{T}\left(x, \omega \mid \gamma^{s}, \delta^{s}\right) .\right. \\
& \left.{ }_{n} C M A B_{j}^{T}\left(x \mid \gamma^{s}, \delta^{s}\right)\right],
\end{aligned}
$$

where ${ }_{n} F_{j}^{T}\left(x, \omega \mid \gamma^{s}, \delta^{s}\right)$ is the lifetime birth probability of $n$ additional children and $T F_{j}^{T}\left(x \mid \gamma^{s}, \delta^{s}\right)$ is the conditional index of total fertility for the above cohort.

An ongoing postponement of fertility affects this overall mean age at birth through two pathways: On one hand, a postponement of fertility at some parity $j+n$ increases the cohort mean age at birth at parity $j+n$. On the other hand, an ongoing delay of childbearing can imply fertility aging effects and therefore shift the final parity distribution in the synthetic cohorts towards lower parities. The overall mean age at birth therefore increases at a slower pace than the parity-specific cohort mean ages.

Unfortunately, the above results do not provide an explicit expression for the effect of an ongoing fertility postponement of the synthetic cohort mean age at birth. In general, 
these effects need to be investigated numerically. For first births, however, we can obtain a formula that relates the pace of a future postponement of fertility to the synthetic cohort mean age at first birth:

Result 15 Comparison of conditional cohort mean age at first birth under different postponement scenarios: Consider the period-T cohort of age $x$ and parity zero. Denote as ${ }_{1} C M A B_{0}^{T}\left(x \mid \gamma^{s}, \delta^{s}=0\right)$ the conditional cohort mean age at first birth in this cohort for the special case when there is only a mean change at parity zero, specified by $\gamma_{0}^{s}$, and no variance change (i.e., $\left.\delta_{0}^{s}=0\right)$. Moreover, denote as ${ }_{1} C M A B_{0}^{T}\left(x \mid \gamma^{s}=0, \delta^{s}=0\right)$ the corresponding cohort mean age at first birth in the postponement stops scenario, i.e., the special case when neither a mean nor variance change occurs after the reference year $\left(\gamma_{0}^{s}=0\right.$ and $\left.\delta_{0}^{s}=0\right)$.

The difference in the cohort mean age at first birth caused by the annual mean change $\gamma_{0}^{s}$ during the life-course of the cohort then follows as

$$
\begin{aligned}
\Delta_{1} C M A B_{0}^{T}(x) & ={ }_{1} C M A B_{0}^{T}\left(x \mid \gamma^{s}, \delta^{s}=0\right)-{ }_{1} C M A B_{0}^{T}\left(x \mid \gamma^{s}=0, \delta^{s}=0\right) \\
& =\frac{\gamma_{0}^{s}}{1-\gamma_{0}^{s}}\left[{ }_{1} C M A B_{0}^{T}\left(x \mid \gamma^{s}=0, \delta^{s}=0\right)-x\right]
\end{aligned}
$$

or equivalently,

$$
{ }_{1} C M A B_{0}^{T}\left(x \mid \gamma^{s}, \delta^{s}=0\right)=\frac{1}{1-\gamma_{0}^{s}}\left[{ }_{1} C M A B_{0}^{T}\left(x \mid \gamma^{s}=0, \delta^{s}=0\right)-x\right]+x .
$$

Moreover, consider childless cohorts who have not entered childbearing in the reference year T. For these cohorts, the cohort mean age at first birth in the postponement stops scenario does not depend on the age $x$ in the reference year, i.e., $\frac{d}{d x}{ }_{1} C M A B_{0}^{T}\left(x \mid \gamma^{s}=\right.$ $\left.0, \delta^{s}=0\right)=0$. If the postponement continues after the reference year, however, these cohorts differ in their mean age at first birth. In particular, the cohort mean age at first birth in the postponement continues scenario changes across cohorts as

$$
\frac{d}{d x}{ }_{1} C M A B_{0,1}^{T}\left(x \mid \gamma^{s}, \delta^{s}=0\right)=-\frac{\gamma_{0}^{s}}{1-\gamma_{0}^{s}}
$$

The above results for first births have several interesting implications: $(a)$ The mean age at birth in cohorts is centrally related to the fraction $\gamma_{0}^{s} /\left(1-\gamma_{0}^{s}\right)$; in particular, the average age at first birth of two cohorts, who have not entered childbearing ages, differs by the product of $\gamma_{0}^{s} /\left(1-\gamma_{0}^{s}\right)$ multiplied with the age-difference between these two cohorts (see Eq. 23; for a related discussion in the context of the Bongaarts-Feeney formula see Zeng Yi and Land 1999). (b) The change in the cohort mean age at first birth caused by an ongoing delay of childbearing depends on the difference between the age of a cohort in the 
reference year $T$ and the conditional cohort mean age at first birth in the postponement stops scenario (see Eq. 21). The greater this difference is, the larger the effect of an ongoing delay of childbearing is. Or alternatively, the effect of a future postponement of first births on the timing of cohort fertility increases the younger the cohort is and the later the timing of childbearing is.

\section{Using the Model: Measuring Period Fertility and Com- pleting Cohort Fertility}

In the previous sections we have completed our development of conditional parity progression measures for the period- $T$ cohort of age $x$ and parity $j$ under alternative postponement scenarios. The two main applications follow directly from this analysis: $(a)$ an improved measurement of period fertility on the basis of period parity progression measures that are free from tempo distortions, and $(b)$ a possibility to complete and project the fertility of birth cohorts, who are still in childbearing ages, under different postponement scenarios.

\subsection{Parity progression measures for measuring period fertility}

The parity progression measures introduced in the previous section can be used for the measurement of period fertility when they are applied to synthetic cohorts. This is particularly meaningful for the postponement stops scenario. In this case, the synthetic cohort experiences the tempo-adjusted period intensities. If we choose a synthetic cohort that is at the beginning of its childbearing ages, this calculation yields the period fertility index $(P F)$. This index represents a tempo-adjusted version of the $P A T F R$ index introduced by Rallu and Toulemon (1994), and it is equal to

$$
P F_{t}=T F_{0}^{t}\left(\alpha \mid \gamma^{s}=0, \delta^{s}=0\right),
$$

where $\alpha$ is the earliest age at childbearing (in our calculations we have used $\alpha=15$ ).

The primary advantage of the period fertility index, $P F_{t}$, is its direct interpretation in terms of synthetic cohort fertility: it is equal to the total fertility of women in a synthetic cohort that experiences the tempo-adjusted childbearing intensities in period $t$ throughout their childbearing ages. This measure is free of tempo and compositional distortions because the calculations are based on tempo-adjusted childbearing intensities instead of incidence rates. The period fertility index, $P F_{t}$, is therefore a period measure of fertility that can be interpreted as the quantum of fertility in period $t$, where quantum is defined as the cohort fertility level associated with the tempo-adjusted childbearing intensities in 
a calendar year (for more extended discussions of measuring period fertility, see Ortega and Kohler 2002a,b).

While the period fertility index reflects a central aspect of period fertility, namely the period quantum of fertility, various other period parity progression measures can also be applied to the synthetic cohort under the postponement stops scenario. For instance, the period lifetime birth probabilities, the period parity progression ratios and the cohort mean age at birth can also be used to characterize synthetic cohort fertility and to describe period patterns.

If these measures are calculated under the postponement stops scenario, they can be regarded as proper period measures of fertility. They are neither affected by compositional distortions, which represent influences of prior fertility behaviors, nor by tempo distortions, which are due to changes in the timing of fertility. In addition to these calculations under the postponement stops scenario, it is often useful to calculate parity progression measures under the postponement continues scenario to illustrate the implications of fertility aging. In particular, an alternative summary index that we will report in our analysis is the index of total fertility for the postponement continues scenario, $T F_{0}^{t}\left(\alpha \mid \gamma^{s}=\gamma(t), \delta^{s}=\delta(t)\right)$. It measures the total fertility of women in a synthetic cohort that experience an ongoing delay of childbearing, at a pace that equals the mean- and variance changes in period $t$, throughout their childbearing years. This measure therefore provides an overall assessment of the net fertility aging effect that is implied by the period $t$ postponement pattern.

\subsection{Completing the fertility of cohorts}

The second application of our period parity progression measures is the completion of cohort fertility. This completion is possible because the measures introduced in the previous section provide conditional projections for the period- $T$ cohorts at all ages and parities. This allows us to take the parity distribution observed in the reference year $T$ as the starting point for the calculation. We then use period parity progression measures to fill in the 'missing' part of a cohort's childbearing pattern conditional on future trends in the tempo and parity-specific level of fertility. In the cohort completions that we present in this paper, the projection of future fertility is based on the parity-specific level of fertility $q_{j}(T)$ in the reference year and the parity-specific postponement pattern given by $\gamma_{j}^{s}$ and $\delta_{j}^{s}$ for all parities $j=0, \ldots$. The completed fertility of birth cohorts then follows via a weighted average of the conditional total fertility indexes $T F_{j}^{T}\left(x \mid \gamma^{s}, \delta^{s}\right)$ across all parities in the reference year $T$, where the weights are equal to the observed parity distribution of the cohort in the reference year $T$. That is, if ${ }_{j} D_{o b s}^{T}(x)$ is the fraction of women at exact parity $j$ among all women who are age $x$ in the reference year $T$, the conditional completed 
fertility of the birth cohort age $x$ in period $T$ is given by

$$
C F^{T}\left(x \mid \gamma^{s}, \delta^{s}\right)=\sum_{j \geq 0}{ }_{j} D_{o b s}^{T}(x) \cdot T F_{j}^{T}\left(x \mid \gamma^{s}, \delta^{s}\right) .
$$

This calculation is conditional on a specific postponement scenario that is represented by ' $\gamma^{s}, \delta^{s}$ ' in the above notation. This postponement scenario can be freely chosen by the analyst. Particularly interesting and appealing for cohort completion, in our opinion, are the postponement stops $\left(\gamma^{s}=0, \delta^{s}=0\right)$ and the postponement continues $\left(\gamma^{s}=\gamma^{T}, \delta^{s}=\right.$ $\left.\delta^{T}\right)$ scenarios.

While the completed cohort fertility is a centrally important indicator of cohort fertility, the analysis with our period parity progression measures is not restricted to this measure. Other cohort fertility measures, such as the final parity distribution or the mean age at birth (overall or at some specific birth order), can also be obtained by taking corresponding weighted averages. For instance, the cohort mean age at birth of order $j$ can be calculated via a weighted average between the mean age of women at order- $j$ births prior to the year $T$ and the order-specific mean age at order- $j$ births that are projected to occur in the future.

It is important to emphasize that the above completions of cohort fertility are not demographic forecasts that account for the uncertainty associated with the future level and postponement of fertility. Our calculations are conditional on a specific postponement scenario and they assume the parity-specific level effects $q_{j}$ that prevail in the reference year $T$. Assessing the uncertainty of cohort fertility requires either simulations with alternative assumptions about the level of fertility and pace of fertility postponement, stochastic time-series models for the level, tempo and tempo changes similar to the methods introduced in Lee (1974, 1993), or expert-based probabilistic approaches as in Lutz et al. (1996). While these assessments of uncertainty are quite compatible with our methods, the detailed discussion of these possibilities is beyond the scope of the present analyses. Despite this limitation, the methods introduced here are very useful because they allow a demographically correct and consistent projection of cohort fertility conditional on a level of fertility and a postponement pattern that is derived from past period trends.

\section{Empirical Implementation}

In the following we briefly discuss the empirical implementation and estimation of the tempo-adjusted period parity progression measures proposed in this paper. $S$-plus programs to perform these calculations, and a more detailed discussion of the estimation procedure, are available online at http://user.demogr.mpg.de/kohler. These calculations 
proceed in three steps. First, we obtain childbearing intensities. Second, we estimate mean- and variance changes in the schedules of period childbearing intensities for each calendar year and compute the adjusted childbearing intensities. Third, we use these tempo-adjusted childbearing intensities to calculate fertility measures for the synthetic cohort.

Age-, period- and parity specific childbearing intensities (or occurrence-exposure rates) are usually not published in standard demographic publications. In general, these intensities need to be calculated on the basis of information about (a) births by age and parity in a calendar year, and $(b)$ a measure of the person years lived by women who are 'at risk' of giving birth to a first, second, third, etc., child. For instance, the exposure can be estimated by the mid-year female population by age and parity. These data can be easily obtained in countries with population registers. The data can also be reconstructed for many other countries that have regular censuses with questions about parity. Another possibility is to obtain childbearing intensities by using data on cohort fertility by age and parity, since age- and parity-specific fertility rates for cohorts can be converted into childbearing intensities. For instance, the Observatoire Démographique Européen has reconstructed such cohort data for many countries (for publications that use these data, see for instance Frejka and Calot 2001a,b or Kohler, Billari, and Ortega 2001).

The second step requires that we calculate the mean and variances of the adjusted intensity schedules in all calendar years. In the presence of variance effects, the respective estimation is somewhat more complicated than in the standard BF adjustment of the total fertility rate because variance effects distort the shape of the intensity schedule. [Note 12] The shape of the observed intensity schedule therefore differs from the adjusted intensity schedule at parity $j$ whenever $\delta_{j}(t) \neq 0$. In order to properly estimate the adjusted intensity schedule along with its mean- and variance changes we therefore implement the following iterative procedure: [Note 13]

1. We estimate the mean age and variance of all period intensity schedules for parities $j=1, \ldots J$, where $J$ is the highest parity. Since childbearing intensities for higher parities tend to be quite variable at young ages, it is advisable to restrict the intensities to 'relevant' ages (for instance, in our analyses for Sweden we have dropped the childbearing intensities for third and fourth births below the ages of 20 and 21 , since third and fourth birth fertility below these ages do not contribute importantly to the overall period and cohort fertility).

2. We estimate the mean and variance changes in the observed childbearing intensities. In order to achieve a robust estimation, we use IRW methods based on state-space smoothing (García-Ferrer et al. 2001; Young 1994), where the ratio of the variance of the noise in the mean age series to the variance in the derivative (the so-called Noise Variance Ratio, $N V R$ ) is fixed. [Note 14] 
3. We estimate the adjusted intensity schedule at parity $j$ on the basis of the observed childbearing intensities and the annual mean- and variance changes obtained in the previous step (see Result 2).

4. We re-estimate the mean- and variance change, $\gamma_{j}(t)$ and $\delta_{j}(t)$, for all calendar years based on the adjusted intensity schedules obtained in the previous step.

5. We return to step 3 and re-estimate the adjusted intensity schedule based on the new values of $\gamma_{j}(t)$ and $\delta_{j}(t)$. The iteration stops once the estimates for the mean- and variance changes converge.

Once the adjusted intensity schedules have been estimated for all calendar years, the tempo-adjusted parity progression measures proposed in this paper can be calculated by using discrete-time versions of our earlier results.

Acknowledgments: We are greatly indebted to Gunnar Andersson for providing us with the Swedish data used in this paper. This paper has also greatly benefited from earlier discussions with Kenneth Wachter. In addition, we have received many very helpful comments and suggestions from two anonymous reviewers, Juha Alho, Francesco Billari, John Bongaarts, Tomas Frejka, Patrick Heuveline, Jan M. Hoem, Evert van Imhoff, Nico Keilman, Ron Lesthaeghe and Laurent Toulemon. We greatly appreciate the support of the Max Planck Institute for Demographic Research, where Ortega has been a guest researcher from June 2001 to January 2002. Ortega has also received financial support from CICYT, program PB98-0075. 


\section{Notes}

1. The alternative specification would have been a parity and duration model (e.g., see Feeney and Yu 1987; Ní Bhrolcháin 1987; Rallu and Toulemon 1994) that does not exhibit any fertility aging effects since childbearing intensities depend exclusively on the duration since the last birth. This implication is contrary to the current empirical evidence, so the parity and age model is preferred on these grounds.

2. As Laurent Toulemon commented in personal communication this is to a large extent a result of the model. If transition to first birth requires earlier transitions out of education or into cohabitation or marriage, these could be accommodated within an extended fertility model as prior states. Postponement of such transitions would have consequences on the ultimate level of childlessness.

3. Toulemon and Mazuy (2001) call this a strong independence hypothesis. Van Imhoff (2001) advocates the use of the postponement stops scenario for the same reasons. We note that if one believes that fertility postponement at lower parities will continue but that the postponement at higher parities will compensate for the fertility aging effect, this is not the same as the postponement stops scenario: while the ultimate level of fertility for a cohort starting childbearing now would coincide, that of actual cohorts would be different since the childbearing intensities would be applied to the wrong cohorts if one believes that postponement will continue. We do not pretend to say ex-ante what the most likely scenario would be; we simply provide the two present scenarios for illustrative purposes. Fertility forecasting as compared to projection requires a modeling exercise that includes an assessment of uncertainty. Such analyses, however, are is beyond the scope of this paper.

4. In this paper we do not systematically address whether the high mean ages at first and higher order births in the postponement continues scenario are realistic given the foreseeable social changes and medical progress in the future. The goal of this paper is to study the implications of postponed childbearing and to provide techniques to construct and study future scenarios about the postponement of fertility. These scenarios can then provide the basis for analyses of the plausibility of these developments, and they can provide the information for studies about the necessary social, demographic and medical changes that are necessary in order to accommodate further delays in childbearing.

5. Several methods and models have been proposed to estimate period parity progression ratios and related measures from vital registration data, survey data, or a combination of 
both (Barkalov and Dorbritz 1996; Chiang and van den Berg 1982; Feeney 1985; Handcock et al. 2000; Henry 1953; Lutz 1989; Ortega and Kohler 2002a; Rallu and Toulemon 1993). The proposed methods differ in their demands for parity-specific data on births and population, and in their assumptions about the determinants of fertility.

6. The requirement that $R_{j}(a, T)=0$ and $\left.\Delta_{a} R_{j}(a, t)\right|_{t=T}=0$ at all ages $a$ is an implication of choosing time $T$ as 'reference year' in the sense that we measure changes in the age pattern of childbearing intensities relative to the age pattern at time $T$. By rescaling the cumulated tempo $R_{j}(a, t)$, the reference year $T$ can be changed without affecting any of the subsequent results of adjusted childbearing intensities. The assumption that $a-R_{j}(a, t)$ is increasing in $a$, or equivalently, that $1-\Delta_{a} R_{j}(a, t)>0$, is a consistency restriction that ensures that the mapping between the observed $h$ schedule and the standard $\phi$ schedule follows the natural age order. See Kohler and Philipov (2001) for a further discussion.

7. See KP for expressions for the mean age and variance of the observed intensity schedule, which differ from those of the adjusted intensity schedule when variance changes are present, i.e., when $\delta_{j}^{s} \neq 0$.

8. In formal analyses, this condition can always be satisfied by choosing $\omega$ sufficiently high.

9. In principle, our theoretical framework is sufficiently general so that any specific assumption about $\gamma_{j}^{s}$ and $\delta_{j}^{s}$, which is deemed relevant and provides a plausible scenario for the future fertility postponement, can be implemented.

10. An alternative to the integral calculations in the Results $9-12$ is the use of a multistate life table program that takes the childbearing intensities $m_{j}^{s}(a, t)$ in Eq. (14) as an input and integrates them along cohort lines; see for instance the LIPRO program by van Imhoff and Keilman (1991).

11. In particular, the childbearing $m_{j}^{s}(a, T+a-x)$ in Eq. (18) can also be obtained from the conditional parity progression probability as

$$
m_{j}^{s}(a, T+a-x)=-\Delta_{a} \log \left(1-p_{j}^{T}\left(x, a \mid \gamma^{s}, \delta^{s}\right)\right),
$$

and the integral in Eq. (18) can then be expressed completely in terms of conditional parity progression probabilities. 
Equation (24) follows as

$$
\begin{aligned}
\Delta_{a} \log \left(1-p_{j}^{T}\left(x, a \mid \gamma^{s}, \delta^{s}\right)\right) & =\Delta_{a}\left[-\int_{x}^{a-R_{j}^{s}(a, T+(a-x))} m_{j}^{\prime}(z, T) d z\right] \\
& =-q_{j}(T)\left(1-\left.\Delta_{a, t} R_{j}^{s}(a, t)\right|_{a, t=T+(a-x)}\right) \phi_{j}^{T}(a) \\
& =-\left(1-\left.\Delta_{a, t} R_{j}^{s}(a, t)\right|_{a, t=T+(a-x)}\right) m_{j}^{\prime}(a, T) \\
& =-m_{j}^{s}(a, T+(a-x))
\end{aligned}
$$

The first equality follows from the definition of $p_{j}^{T}\left(x, a \mid \gamma^{s}, \delta^{s}\right)$ in Eq. (17), the second equality follows by differentiation, the third equality follows by inserting $m_{j}^{\prime}(a, T)=$ $q_{j}(T) \phi_{j}^{T}(a)$ (see Definition 2), and the final equality follows from the definition of $m_{j}^{s}(a$, $t)$ in Eq. (14).

12. See Kohler and Philipov (2001) for a detailed discussion of the distortions in the observed fertility pattern that are caused by variance effects

13. This procedure is slightly different from the one implemented in Kohler and Philipov (2001) and provides estimates of the adjusted intensity schedules and not only the moments of these schedules as in KP.

14. The model can be written as follows. The measurement equation states that the observed series (in our case the mean age and standard deviation of the period intensity schedule at parity $j$ ) is the sum of an unobserved trend component, $T_{t}$, and a noise component with variance $\sigma_{\varepsilon}^{2}$ :

$$
Y_{t}=T_{t}+\varepsilon_{t}
$$

The transition equations state that the trend component changes in each period through the addition of a slope component (the derivative estimate) which changes over time through the addition of a noise component $\eta_{t}$ with variance $\sigma_{\eta}^{2}$ :

$$
\begin{aligned}
T_{t} & =T_{t-1}+S_{t-1} \\
S_{t} & =S_{t-1}+\eta_{t}
\end{aligned}
$$

The system depends on a single parameter, the noise variance ratio $N V R=\sigma_{\varepsilon}^{2} / \sigma_{\eta}^{2}$, that reflects the variance of noise in the observed series $Y_{t}$ relative to the noise in the underlying trend component $T_{t}$. The extraction of the trend and slope components implies the forward application of the Kalman filter algorithm and the backward application of Fixed Interval Smoothing. We implement this smoothing technique because of its desirable spectral properties: there is a relationship between the $N V R$ and the cut-off frequency of the filter. 


\section{References}

Andersson, G. (1999). Childbearing trends in Sweden 1961-1995. European Journal of Population 15(1), 1-24.

Andersson, G. (2000). The impact of labor-force participation on childbearing behavior: Pro-cyclical fertility in Sweden during the 1980s and the 1990s. European Journal of Population 16(4), 293-333.

Andersson, G. and L. Guiping (2001). Demographic trends in Sweden: Childbearing developments in 1961-1999, marriage and divorce developments in 1971-99. Demographic Research [online available at http://www.demographic-research.org] 5(3), 65-78.

Barkalov, N. B. and J. Dorbritz (1996). Measuring period parity-progression ratios with competing techniques: An application to East Germany. Zeitschrift für Bevölkerungswissenschaft 21(4), 459-505.

Billari, F. C. and H.-P. Kohler (2002). The impact of union formation dynamics on first births in West Germany and Italy: Are there signs of convergence? In E. Klijzing and M. Corijn (Eds.), Fertility and Partnership in Europe: Findings and Lessons from Comparative Research, Volume 2, forthcoming. Geneva / New York: United Nations.

Bongaarts, J. and G. Feeney (1998). On the quantum and tempo of fertility. Population and Development Review 24(2), 271-291.

Brass, W. (1990). Cohort and time period measure of quantum and fertility: Concepts and methodology. In H. A. Becker (Ed.), Life Histories and Generations, Volume 2, pp. 455-476. Utrecht: ISOR, Faculty of Social Sciences, University of Utrecht.

Bumpass, L. L. and E. K. Mburugu (1977). Age at marriage and completed family size. Social Biology 24(1), 31-37.

Bumpass, L. L., R. R. Rindfuss, and R. B. Janosik (1978). Age and marital status at first birth and the pace of subsequent fertility. Demography 15(1), 75-86.

Chiang, C. L. and B. van den Berg (1982). A fertility table for the analysis of human reproduction. Mathematical Biosciences 62(2), 237-251.

Council of Europe (1999). Recent Demographic Developments in Europe. Strasbourg: Council of Europe Publishing. 
Feeney, G. (1985). Parity-progression projection. In International Population Conference, Florence 1985, Volume 4, pp. 125-136. Liége, Belgium: International Union for the Scientific Study of Population.

Feeney, G. and W. Lutz (1991). Distributional analysis of period fertility. In W. Lutz (Ed.), Future Demographic Trends in Europe and North America: What Can We Assume Today?, pp. 169-196. London: Academic Press.

Feeney, G. and J. Yu (1987). Period parity progression measures of fertility in China. Population Studies 41(1), 77-102.

Frejka, T. and G. Calot (2001a). Cohort reproductive patterns in low-fertility countries. Population and Development Review 27(1), 103-132.

Frejka, T. and G. Calot (2001b). L'évolution du calendrier des naissances par génération dans les pays à basse fécondité à la fin du XXe siècle. Population 56(3), 397-420.

García-Ferrer, A., R. Queralt, and C. Blázquez (2001). A growth cycle characterisation and forecasting of the Spanish economy: 1970-1998. International Journal of Forecasting 17, 517-532.

Hajnal, J. (1947). Aspects of recent trends in marriage in England and Wales. Population Studies 1(1), 72-92.

Hajnal, J. (1953). Age at marriage and proportions marrying. Population Studies 7(2), $111-136$.

Handcock, M. S., S. M. Huovilainen, and M. S. Rendall (2000). Combining registrationsystem and survey data to estimate birth probabilities. Demography 37(2), 187-192.

Henry, L. (1953). Fécondité des mariages: nouvelle méthode de mesure. Paris. English Translation: Fertility of Marriage: A New Method of Measurement, UN/ESCAP Population Studies Translation Series No. 3, 1980.

Henry, L. (1972). Démographie: Analyse et Modèles. Paris: Librairie Larousse.

Hoem, B. (2000). Entry into motherhood in Sweden: The influence of economic factors on the rise and fall in fertility, 1986-1997. Demographic Research [online available at http://www.demographic-research.org] 2(4).

Hoem, B. and J. M. Hoem (1996). Sweden's family policies and roller-coaster fertility. Jinko Mondai Kenkyu / Journal of Population Problems 52(3-4), 1-22. 
Hoem, J. M. (1990). Social policy and recent fertility change in Sweden. Population and Development Review 16(4), 735-748.

Hoem, J. M. and U. F. Jensen (1982). Multistate life table methodology: A probabilistic critique. In K. C. Land and A. Rodgers (Eds.), Multidimensional Mathematical Demography, pp. 155-264. New York: Academic Press.

Keilman, N. (1994). Translation formulae for non-repeatable events. Population Studies 48(2), 341-357.

Keilman, N. and E. van Imhoff (1995). Cohort quantum as a function of time-dependent period quantum for non-repeatable events. Population Studies 49(2), 347-352.

Kim, Y. J. and R. Schoen (2000). On the quantum and tempo of fertility: Limits to the Bongaarts-Feeney adjustment. Population and Development Review 26(3), 554-559.

Kohler, H.-P., F. C. Billari, and J. A. Ortega (2001). Towards a theory of lowest-low fertility. Max Planck Institute for Demographic Research, Rostock, Germany, Working Paper \#2001-032 (available at http://www.demogr.mpg.de).

Kohler, H.-P. and J. A. Ortega (2002). Tempo-adjusted period parity progression measures: Assessing the implications of delayed childbearing for fertility in Sweden, the Netherlands and Spain. Demographic Research [online available at http:// www.demographic-research.org] 6(7), 145-190.

Kohler, H.-P. and D. Philipov (2001). Variance effects in the Bongaarts-Feeney formula. Demography 38(1), 1-16.

Kohler, H.-P., A. Skytthe, and K. Christensen (2001). The age at first birth and completed fertility reconsidered: Findings from a sample of identical twins. Max Planck Institute for Demographic Research, Rostock, Germany, Working Paper \#2001-006 (available at http://www.demogr.mpg.de).

Le Bras, H. (1997). Fertility: The condition of self-perpetuation. Differing trends in Europe. In M. Gullestad and M. Segalen (Eds.), Family and Kinship in Europe, pp. 14-32. Pinter Publishers.

Lee, R. D. (1974). Forecasting births in post-transition populations: Stochastic renewal with serially correlated fertility. Journal of the American Statistical Association 69(347), 607-617.

Lee, R. D. (1993). Modeling and forecasting the time series of U.S. fertility: Age distribution, range and ultimate level. International Journal of Forecasting 9(2), 187-202. 
Lotka, A. J. and M. Spiegelman (1940). The trend of the birth rate by age of mother and order of birth. Journal of the American Statistical Association 35(212), 595-601.

Lutz, W. (1989). Distributional Aspects of Human Fertility: A Global Comparative Study. New York: Academic Press.

Lutz, W., W. Sanderson, and S. Scherbov (1996). Probabilistic population projections based on expert opinions. In W. Lutz (Ed.), The Future Population of the World: What Can We Assume Today?, pp. 397-428. Earthscan Publications.

Marini, M. M. and P. J. Hodsdon (1981). Effects of the timing of marriage and first birth on the spacing of subsequent births. Demography 18(4), 529-548.

Morgan, P. S. and R. R. Rindfuss (1999). Reexamining the link of early childbearing to marriage and to subsequent fertility. Demography 36(1), 59-75.

Ní Bhrolcháin, M. (1987). Period parity progression ratios and birth intervals in England and Wales, 1941-1971: A synthetic life table analysis. Population Studies 41(1), 103125 .

Ní Bhrolcháin, M. (1992). Period paramount? A critique of the cohort approach to fertility. Population and Development Review 18(4), 599-629.

Ortega, J. A. and H.-P. Kohler (2002a). Measuring low fertility: Rethinking demographic methods. Max Planck Institute for Demographic Research, Rostock, Germany, Working Paper \#2002-001 (available at http://www.demogr.mpg.de).

Ortega, J. A. and H.-P. Kohler (2002b). A new look at period fertility: A decomposition of the effects of parity distribution, tempo distortions and fertility on the period TFR. Mimeo, Max Planck Institute for Demographic Research, Rostock, Germany.

Park, C. B. (1967). Measuring the probability of eventually bearing $n$ live births: An extension of fertility tables. Proceedings of the American Statistical Association, Social Science Section, 1967, 38-49.

Park, C. B. (1976). Lifetime probability of additional births by age and parity for American women, 1935-1968: A new measurement of period fertility. Demography 13(1), $1-17$.

Presser, H. B. (1971). The timing of the first birth, female roles and black fertility. Milbank Memorial Fund Quarterly 49, 329-362.

Quensel, C.-E. (1939). Changes in fertility following birth restriction. Skandinavisk Aktuarietidskrift 22(3-4), 177-199. 
Rallu, J.-L. and L. Toulemon (1993). Les mesures de la fécondité transversale I. Construction des differénts indices. Population 48(1), 7-26.

Rallu, J.-L. and L. Toulemon (1994). Period fertility measures: The construction of different indices and their application to France, 1946-89. Population: An English Selection 6, 59-130.

Ryder, N. B. (1964). The process of demographic translation. Demography 1(1), 74-82.

Ryder, N. B. (1983). Cohort and period measures of changing fertility. In R. Bulatao and R. D. Lee (Eds.), Determinants of Fertility in Developing Countries, Volume 2, pp. 737-756. New York: Academic Press.

Schoen, R. (1988). Modeling Multigroup Populations. New York: Plenum.

Toulemon, L. and M. Mazuy (2001). Cinq projections de fécondité fondées sur une hypothèse de stabilité des comportements. Population 56(4), 647-656.

Trussell, J. and J. Menken (1978). Early childbearing and subsequent fertility. Family Planning Perspectives 10(4), 209-218.

van Imhoff, E. (2001). On the impossibility of inferring cohort fertility measures from period fertility measures. Demographic Research [online available at http:// www.demographic-research.org] 5(2), 23-64.

van Imhoff, E. and N. Keilman (1991). LIPRO 2.0: An Application of a Dynamic Demographic Projection Model to Household Structure on the Netherlands. Amsterdam/Lisse: Swets \& Zeitlinger. (latest available version: LIPRO 4.0).

van Imhoff, E. and N. Keilman (2000). On the quantum and tempo of fertility: Comment. Population and Development Review 26(3), 549-553.

Whelpton, P. K. (1946). Reproduction rates adjusted for age, parity, fecundity, and marriage. Journal of the American Statistical Association 41(236), 501-516.

Young, P. C. (1994). Time variable parameter and trend estimation in non-stationary economic time series. Journal of Forecasting 13(2), 179-210.

Zeng Yi and K. Land (1999). Ryder's basic translation equation revisited and an adjustment for bias in observed changes in period tempo of demographic events. Unpublished manuscript, Center for Demographic Studies, Duke University, Durham. 


\section{Appendix: Proofs omitted in the text}

Proof of Result 1: The relation between the two schedules $h_{j}(a, t)$ and $\phi_{j}^{T}(a)$ can be established by a change of variable from the observed age $a$ and time $t$ of a birth at parity $j$ to the hypothetical age $a-R_{j}^{T}(a, t)$ and time $t-R_{j}^{T}(a, t)$ at which this birth would occur in the absence of any further fertility postponement after the reference year $T$. The Jacobian of this transformation from the observed to hypothetical occurrence of a birth is $\left(1-\Delta_{a, t} R_{j}^{T}(a, t)\right)$. The relation between $h_{j}(a, t)$ and $\phi_{j}^{T}(a)$ therefore is given by

$$
h_{j}(a, t)=\left(1-\Delta_{a, t} R_{j}^{T}(a, t)\right) \cdot \phi_{j}^{T}\left(a-R_{j}^{T}(a, t)\right) .
$$

Moreover, the above childbearing intensities $h_{j}(a, t)$ in Eq. (26) satisfy our definition of fertility postponement (see Eq. 5 in Definition 1) for all choices of $x$ and $y$. In particular, the equality of the probabilities $p_{j, T}^{h}(x, y)$ and $p_{j, T}^{\phi}(x, z)$ in Definition 1 is obtained by integrating

$$
\begin{aligned}
& p_{j, T}^{h}(x, y) \\
= & 1-\exp \left[-\int_{x}^{y} h_{j}(a, T+(a-x)) d a\right] \\
= & 1-\exp \left[-\int_{x}^{y}\left(1-\left.\Delta_{a, t} R_{j}^{T}(a, t)\right|_{a, t=T+(a-x)}\right) \cdot \phi_{j}^{T}\left(a-R_{j}^{T}(a, T+(a-x))\right) d a\right] \\
= & 1-\exp \left[-\int_{x}^{y-R(y, T+(y-x))} \phi_{j}^{T}(\alpha) d \alpha\right] \\
= & p_{j, T}^{\phi}(x, z) \quad \text { with } z=y-R\left(y, T^{\prime}\right) \text { and } T^{\prime}=T+(y-x) .
\end{aligned}
$$

The first equality follows from the definition of $p_{j, T}^{h}(x, y)$ in Eq. (3). The second equality follows by inserting Eq. (26) and substituting $t=T+(a-x)$, where we have used the notation ' $\left.\right|_{a, t=T+(a-x)}$ ' to indicate that the derivative $\Delta_{a, t} R_{j}^{T}(a, t)$ is evaluated at age $a$ and time $t=T+(a-x)$. The third equality follows by changing the variable of integration from the age $a$ to the hypothetical age $\alpha=a-R_{j}^{T}(a, T+(a-x))$ at which births would have occurred in the absence of a fertility postponement. The Jacobian that needs to be considered in this change of variable is $\left(1-\left.\Delta_{a, t} R_{j}^{T}(a, t)\right|_{a, t=T+(a-x)}\right)^{-1}$. This Jacobian cancels the first term within the integral sign. Moreover, the cumulated tempo change evaluated in the reference year $T, R_{j}^{T}(a, T)$, is equal to zero at all ages (Assumption 4) and there is no change in the lower limit of the integral after the change of variable. The final equation follows directly from the definition of $p_{j, T}^{\phi}(x, z)$ in Eq. (4) after inserting $z=y-R\left(y, T^{\prime}\right)$ and $T^{\prime}=T+(y-x)$.

The above steps therefore establish for all values of $x$ and $y$ the equality $p_{j, T}^{h}(x, y)=$ $p_{j, T}^{\phi}(x, z)$ with $z=y-R\left(y, T^{\prime}\right)$ and $T^{\prime}=T+(y-x)$. Hence, the observed age-pattern 
of childbearing intensities given by Eq. (26) imply a postponement of fertility according to Definition 1 , and vice versa.

In combination with decomposition of the observed childbearing intensities in Eq. (2), the result in Eq. (26) also yields the relation for the observed childbearing intensities in Eq. (7).

Proof of Result 2: The result follows from the specification of the adjusted childbearing intensities in Definition 2 and by integrating $\int m_{j}^{\prime}(a, t) d a=q_{j}(t) \int\left(1-\Delta_{a} R_{j}^{T}(a, t)\right)$ $\phi_{j}^{T}\left(a-R_{j}^{T}(a, t)\right) d a$. A change of variable from $a$ to $\alpha=a-R_{j}^{T}(a, t)$ then yields $\int m_{j}^{\prime}(a, t) d a=q_{j}(t) \int \phi_{j}^{T}(\alpha) d \alpha=q_{j}(t)$, where the last equality follows from Assumption 3 .

Proof of Result 3: Result 3 follows directly by rewriting the Jacobian term in Eq. (7) as $\left(1-\Delta_{a, t} R_{j}^{T}(a, t)\right)=\left(1-r_{j}(a, t)\right) \cdot\left(1-\Delta_{a} R_{j}^{T}(a, t)\right)$, where $r_{j}(a, t)$ is defined in Eq. (10).

Proof of Result 4: Cumulated tempo changes must be additive over time. We therefore consider an arbitrary age $a^{\prime}$ and time $t^{\prime}$, where we assume for simplicity that $T<T^{\prime} \leq t^{\prime}$ (different sequences of $T, T^{\prime}$ and $t^{\prime}$ follow analogously), and we decompose the cumulated tempo changes over time. In particular, in the case $T<T^{\prime} \leq t^{\prime}$, the relation

$$
R_{j}^{T}\left(a^{\prime}, t^{\prime}\right)=R_{j}^{T^{\prime}}\left(a^{\prime}, t^{\prime}\right)+R_{j}^{T}\left(a^{\prime}-R^{T^{\prime}}\left(a^{\prime}, t^{\prime}\right), T^{\prime}\right),
$$

which must be satisfied by the cumulated tempo changes for the reference years $T$ and $T^{\prime}$ according to Result 4, follows by separately considering and then adding the cumulated tempo changes between $T$ and $T^{\prime}$, and between $T$ and $t^{\prime}$ :

First, $R_{j}^{T}\left(a^{\prime}, t^{\prime}\right)$ equals to the cumulated tempo changes at age $a^{\prime}$ and time $t^{\prime}$ measured since the reference year $T$. Second, $R_{j}^{T^{\prime}}\left(a^{\prime}, t^{\prime}\right)$ is the corresponding cumulated tempo change measured since the reference year $T^{\prime}$. Third, denote as $\alpha^{\prime}=a^{\prime}-R_{j}^{T^{\prime}}\left(a^{\prime}, t^{\prime}\right)$ the age when a birth occurring at age $a^{\prime}$ and time $t^{\prime}$ would have occurred if there had been no further tempo changes since the reference year $T^{\prime}$. Then $R_{j}^{T}\left(\alpha^{\prime}, T^{\prime}\right)$ represents exactly that part of the overall cumulated tempo change $R_{j}^{T}\left(a^{\prime}, t^{\prime}\right)$ that occurs between the old and new reference years, $T$ and $T^{\prime}$. Adding these contributions then yields $R_{j}^{T}\left(a^{\prime}, t^{\prime}\right)=R_{j}^{T}\left(\alpha^{\prime}, T^{\prime}\right)+R_{j}^{T^{\prime}}\left(a^{\prime}, t^{\prime}\right)$, which in turn yields Eq. (27) after inserting $\alpha^{\prime}=a^{\prime}-R_{j}^{T^{\prime}}\left(a^{\prime}, t^{\prime}\right)$. This new cumulated tempo also satisfies the condition in Assumption 3 that $\left.R_{j}^{T^{\prime}}(a, t)\right|_{t=T^{\prime}}=0$.

The standardized schedule for the new reference year $T^{\prime}$,

$$
\phi_{j}^{T^{\prime}}(a)=\left(1-\left.\Delta_{a} R_{j}^{T}(a, t)\right|_{t=T^{\prime}}\right) \phi_{j}^{T}\left(a-R_{j}^{T}\left(a, T^{\prime}\right)\right),
$$


is obtained by evaluating Eq. (8) at time $T^{\prime}$ and then dividing by the level effect $q_{j}\left(T^{\prime}\right)$ in the new reference year. An integration with change of variable also shows that $\phi_{j}^{T^{\prime}}(a)$ satisfies the condition in Assumption 3 that $\int \phi_{j}^{T^{\prime}}(a) d a=1$.

Moreover, applying the implicit function theorem to Eq. (27) yields

$$
\begin{aligned}
\left.\Delta_{a} R_{j}^{T^{\prime}}(a, t)\right|_{a=a^{\prime}, t=t^{\prime}} & =\frac{\left.\Delta_{a} R^{T}(a, t)\right|_{a=a^{\prime}, t=t^{\prime}}-\left.\Delta_{a} R^{T}(a, t)\right|_{a=a^{\prime}-R_{j}^{T^{\prime}}\left(a^{\prime}, t^{\prime}\right), t=T^{\prime}}}{1-\left.\Delta_{a} R^{T}(a, t)\right|_{a=a^{\prime}-R_{j}^{T^{\prime}}\left(a^{\prime}, t^{\prime}\right), t=T^{\prime}}} \\
\left.\Delta_{t} R_{j}^{T^{\prime}}(a, t)\right|_{a=a^{\prime}, t=t^{\prime}} & =\frac{\left.\Delta_{t} R^{T}(a, t)\right|_{a=a^{\prime}, t=t^{\prime}}}{1-\left.\Delta_{a} R^{T}(a, t)\right|_{a=a^{\prime}-R_{j}^{T^{\prime}}\left(a^{\prime}, t^{\prime}\right), t=T^{\prime}}} .
\end{aligned}
$$

Equation (29) implies that $a-R_{j}^{T^{\prime}}(a, t)$ is increasing in $a$ as is required by Assumption 3. Moreover, the above relations (30-29) allow us to verify that $R_{j}^{T^{\prime}}(a, t)$, combined with the standardized schedule $\phi_{j}^{T^{\prime}}$, generates the same sequence of tempo-adjusted intensity schedules. In particular, from Definition 2 we obtain

$$
\begin{aligned}
m_{j}^{\prime}\left(a^{\prime}, t^{\prime}\right)= & q_{j}\left(t^{\prime}\right) \cdot\left(1-\left.\Delta_{a} R_{j}^{T^{\prime}}(a, t)\right|_{a=a^{\prime}, t=t^{\prime}}\right) \cdot \phi_{j}^{T^{\prime}}\left(a^{\prime}-R_{j}^{T^{\prime}}\left(a^{\prime}, t^{\prime}\right)\right) \\
= & q_{j}\left(t^{\prime}\right) \cdot\left(1-\left.\Delta_{a} R_{j}^{T}(a, t)\right|_{a=a^{\prime}, t=t^{\prime}}\right) \cdot \\
& \phi_{j}^{T}\left(a^{\prime}-R_{j}^{T^{\prime}}\left(a^{\prime}, t^{\prime}\right)-R_{j}^{T}\left(a^{\prime}-R_{j}^{T^{\prime}}\left(a^{\prime}, t^{\prime}\right), T^{\prime}\right)\right) \\
= & q_{j}\left(t^{\prime}\right) \cdot\left(1-\left.\Delta_{a} R_{j}^{T}(a, t)\right|_{a=a^{\prime}, t=t^{\prime}}\right) \cdot \phi_{j}^{T}\left(a^{\prime}-R_{j}^{T}\left(a^{\prime}, t^{\prime}\right)\right)
\end{aligned}
$$

where the first equality follows from the definition of $m_{j}^{\prime}\left(a^{\prime}, t^{\prime}\right)$ in terms of the reference year $T^{\prime}$, the second equality follows from inserting Eqs. (28) and (29), and the third equality follows from Eq. (27). The last line, however, is identical to Eq. (8) in Definition 2 , and the model therefore defines the same tempo adjusted childbearing intensities independent of the choice of the reference years. This immediately implies that Result 2 holds independent of the choice of reference year. Moreover, using Eqs. (29-30), we can also show that the definition of $r_{j}(a, t)$ in Eq. (10) does not depend on the choice of reference year, and thus Result 3 also holds independent of the specification of the reference year.

Proof of Result 5: The result follows by integrating $\bar{a}_{j}(t)=\int a \cdot m_{j}^{\prime}(a, t) d a / q_{j}(t)$ and $s_{j}^{2}(t)=\int\left(a-\bar{a}_{j}(t)\right)^{2} \cdot m_{j}^{\prime}(a, t) d a / q_{j}(t)$, and changing the variable of integration. The steps are identical to the proof of Results 6 and 10 in KP.

Proof of Result 6: The assumptions about the level and postponement of fertility underlying the intensity schedules $m_{j}^{s}(a, t)$ imply for all $t \geq T$ that $q_{j}(t)=q_{j}(T), \gamma_{j}(t)=\gamma_{j}^{s}$, and $\delta_{j}(t)=\delta_{j}^{s}$. The cumulated tempo $R_{j}^{s}(a, t)$ and the age-specific tempo change $r_{j}^{s}(a, t)$ 
then follow from Assumption 5 with $\gamma_{j}(t)=\gamma_{j}^{s}$ and $\delta_{j}(t)=\delta_{j}^{s}$. Equation (14) then follows directly from Eq. (7) after inserting the above assumptions about the determinants of the childbearing intensities for the synthetic cohort. Equation (16) then follows directly from Eqs. (14) and (9).

The statements about the mean and variance of the adjusted intensity schedule $m_{j}^{s \prime}(a, t)$ follow directly from Result 5 . The statement $\int m_{j}^{s}(a, t) d a=\left(1-\gamma_{j}^{s}\right) \cdot q_{j}(T)$ follows by an analogous integration as in the proof of Results 7 and 10 in KP, and the relation $\int m_{j}^{s \prime}(a, t) d a=q_{j}(T)$ follows directly from Result 2 .

Proof of Result 7: Consider the woman specified in Result 7. The probability that this parity- $j$ woman has another child and progresses to parity $j+1$ follows as

$$
\begin{aligned}
& p_{j}^{T}\left(x, y \mid \gamma^{s}, \delta^{s}\right) \\
= & 1-\exp \left[-\int_{x}^{y} m_{j}^{s}(a, T+(a-x)) d a\right] \\
= & 1-\exp \left[-q_{j}(T) \int_{x}^{y}\left(1-\left.\Delta_{a, t} R_{j}^{s}(a, t)\right|_{a, t=T+(a-x)}\right) .\right. \\
& \left.\phi_{j}^{T}\left(a-R_{j}^{s}(a, T+(a-x))\right) d a\right] \\
= & 1-\exp \left[-q_{j}(T) \int_{x}^{y-R_{j}^{s}(y, T+(y-x))} \phi_{j}^{T}(\alpha) d \alpha\right] \\
= & 1-\exp \left[-\int_{x}^{y-R_{j}^{s}(y, T+(y-x))} m_{j}^{\prime}(\alpha, T) d \alpha\right] .
\end{aligned}
$$

The first equality follows from the definition of $p_{j}^{T}\left(x, y \mid \gamma^{s}, \delta^{s}\right)$ and standard eventhistory analysis. The second equality follows by inserting Eq. (14) and using the notation ' $\left.\right|_{a, t=T+(a-x)}$ ' to indicate that the derivative $\Delta_{a, t} R_{j}^{T}(a, t)$ is evaluated at age $a$ and time $t=T+(a-x)$. The third equality follows by changing the variable of integration from the age $a$ to the hypothetical age $\alpha=\alpha-R_{j}^{T}(a, T+(a-x))$ at which births would have occurred in the absence of a fertility postponement. The Jacobian that needs to be considered in this change of variable is $\left(1-\left.\Delta_{a, t} R_{j}^{T}(a, t)\right|_{a, t=T+(a-x)}\right)^{-1}$, and this Jacobian cancels the first term within the integral sign. Moreover, the cumulated tempo change evaluated in the reference year $T, R_{j}^{T}(a, T)$, is equal to zero at all ages (Assumption 4) and there is no change in the lower limit of the integral after the change of variable. The final equality follows since $m_{j}^{\prime}(a, T)=q_{j}(T) \cdot \phi_{j}^{T}(a)$ (see Assumption 3). Finally, changing the notation for the variable of integration yields Eq. (17) in Result 7.

The conditional lifetime parity progression probability is given by

$$
p_{j}^{T}\left(x, \omega \mid \gamma_{j}^{s}, \delta_{j}^{s}\right)=1-\exp \left[-\int_{x}^{\omega-R_{j}^{s}(\omega, T+(\omega-x))} m_{j}^{\prime}(a, T) d a,\right.
$$


where the upper limit of integration can be changed to $\infty$ since all adjusted childbearing intensities $m_{j}^{\prime}(a, T)$ above ages $\omega-R_{j}^{s}(\omega, T+(\omega-x)$ are equal to zero (this implied by Assumption 6).

Proof of Result 8: Denote as $\tilde{p}_{j}^{T}\left(x, y \mid \gamma^{s}, \delta^{s}\right)$ the conditional parity progression probability that is obtained from the calculation in Eq. (17) when the observed childbearing intensities $m_{j}(a, T)$, instead of the adjusted childbearing intensities $m_{j}^{\prime}(a, T)$, are used in the integration. The error that is incurred by using the observed instead of the adjusted childbearing intensities then follows from the fact that the observed and adjusted childbearing intensities at time $T$ are related as $m_{j}(a, T)=\left(1-r_{j}(a, T)\right) \cdot m_{j}^{\prime}(a, T)$, where $r_{j}(a, T)$ is given in Eq. (13). Then $\tilde{p}_{j}^{T}\left(x, y \mid \gamma^{s}, \delta^{s}\right)=1-\exp \left[-\int_{x}^{y-R_{j}^{s}(x, T+(y-x))} m_{j}(a, T) d a\right]=$ $1-\exp \left[-\int_{x}^{y-R_{j}^{s}(x, T+(y-x))}\left(1-r_{j}(a, T)\right) m_{j}^{\prime}(a, T)\right] d a$. Using Eq. (13) and denoting $\tilde{y}=y-R_{j}^{s}(x, T+(y-x))$, the exponential term can be further decomposed as

$$
\begin{aligned}
& \left(1-\tilde{p}_{j}^{T}\left(x, y \mid \gamma^{s}, \delta^{s}\right)\right) \\
= & \exp \left[-\int_{x}^{\tilde{y}}\left(1-r_{j}(a, T)\right) \cdot m_{j}^{\prime}(a, T)\right] d a \\
= & \exp \left[-\int_{x}^{\tilde{y}}\left(1-\gamma_{j}(T)-\delta_{j}(T)\left(a-\bar{a}_{j, T}\right)\right) \cdot m_{j}^{\prime}(a, T) d a\right] \\
= & \exp \left[-\left(1-\gamma_{j}(T)\right) \int_{x}^{\tilde{y}} m_{j}^{\prime}(a, T) d a+\delta_{j}(T) \int_{x}^{\tilde{y}}\left(a-\bar{a}_{j, T}\right) \cdot m_{j}^{\prime}(a, T) d a\right] \\
= & \left(1-p_{j}^{T}\left(x \mid \gamma^{s}, \delta^{s}\right)\right)^{1-\gamma_{j}(T)} \cdot \exp \left[\delta_{j}(T) \int_{x}^{\tilde{y}}\left(a-\bar{a}_{j, T}\right) \cdot m_{j}^{\prime}(a, T) d a\right] .
\end{aligned}
$$

The calculation of the conditional parity progression probability based on the observed intensities is therefore distorted. In the special case when $\delta_{j}(T)=0$, the terms involving $\delta_{j}(T)$ vanish and the calculation of the conditional parity progression probability based on the observed childbearing intensities yields $\tilde{p}_{j}^{T}\left(x, y \mid \gamma^{s}, \delta^{s}\right)=1-(1-$ $\left.p_{j}^{T}\left(x, y \mid \gamma^{s}, \delta^{s}\right)\right)^{1-\gamma_{j}(T)}$. Moreover, since $\bar{a}_{j, T}$ is defined as the mean age of the intensity schedule $m_{j}^{\prime}(a, T)$, the integral in the last term for the calculation of the lifetime probability satisfies $\int_{x}^{\tilde{\omega}}\left(a-\bar{a}_{j, T}\right) m_{j}^{\prime}(a, T) d a \geq 0$, where $\tilde{\omega}=\omega-R_{j}^{s}(x, T+(\omega-x))$ and all adjusted intensities $m_{j}^{\prime}(a, T)$ are equal to zero for $a \geq \tilde{\omega}$ (implied by Assumption 6). For $\gamma_{j}(T)>0$ and $\delta_{j}(T) \geq 0$ we therefore obtain $\left(1-\tilde{p}_{j}^{T}\left(x, \omega \mid \gamma^{s}, \delta^{s}\right)\right)>$ $\left(1-p_{j}^{T}\left(x, \omega \mid \gamma^{s}, \delta^{s}\right)\right)$, or equivalently $\tilde{p}_{j}^{T}\left(x, \omega \mid \gamma^{s}, \delta^{s}\right)<p_{j}^{T}\left(x, \omega \mid \gamma^{s}, \delta^{s}\right)$. The correct value of $p_{j}^{T}\left(x, \omega \mid \gamma^{s}, \delta^{s}\right)$ is thus underestimated when the calculation of this conditional parity progression probability in Eq. (17) is based on the observed instead of the adjusted childbearing intensities. 
Proof of Result 9: Consider a woman in the period- $T$ synthetic cohort of age $x$ and parity $j$ specified in Result 9. The relation for $s_{j+n}^{T}\left(a, y \mid \gamma_{j+n}^{s}, \delta_{j+n}^{s}\right)$ in terms of conditional parity progression probabilities follows directly from the integral for the survival probability and the definition of $p_{j}^{T}\left(x, y \mid \gamma_{j}^{s}, \delta_{j}^{s}\right)$. The parity distribution ${ }_{0} D_{j}^{T}\left(x, y \mid \gamma^{s}, \delta^{s}\right)$ then just follows from the probability of 'surviving' in parity $i$ from age $x$ until age $y$ as ${ }_{0} D_{j}^{T}\left(x, y \mid \gamma^{s}, \delta^{s}\right)=s_{j}^{T}\left(x, y \mid \gamma_{j}^{s}, \delta_{j}^{s}\right)=1-p_{j}^{T}\left(x, y \mid \gamma_{j}^{s}, \delta_{j}^{s}\right)$.

The parity distribution ${ }_{n} D_{j}^{T}\left(x, y \mid \gamma^{s}, \delta^{s}\right)$, with $n>0$, follows by integrating the survival probability $s_{j+n}^{T}\left(a, y \mid \gamma^{s}, \delta^{s}\right)$ multiplied by the probability of attaining parity $j+n$ at age $a$. This latter probability is given by ${ }_{n-1} D_{j}^{T}\left(x, a \mid \gamma^{s}, \delta^{s}\right) \cdot m_{j+n-1}^{s}(a, T+$ $a-x)$, where ${ }_{n-1} D_{j}^{T}\left(x, a \mid \gamma^{s}, \delta^{s}\right)$ is the probability of being at exact parity $j+n-1$ at age $a$ and $m_{j+n-1}^{s}(a, T+a-x)$ is the 'hazard' of experiencing a birth of order $j+n$ at age $a$.

Proof of Result 10: The result follows directly from Result 9 by setting $y=\omega$, where $\omega$ is the maximum feasible age at childbirth.

Proof of Result 11: Consider a woman in the period- $T$ synthetic cohort of age $x$ and parity $j$ specified in Result 11 . The birth probability ${ }_{n} F_{j}^{T}\left(x, y \mid \gamma^{s}, \delta^{s}\right)$ follows by integrating from age $x$ to age $y$ the probability of being at exact parity $j+n-1$ at some age $a$, given by ${ }_{n-1} D_{j}^{T}\left(x, a \mid \gamma^{s}, \delta^{s}\right)$, multiplied by the hazard of experiencing a birth of order $j$ at age $a$, given by $m_{j+n-1}^{s}(a, T+a-x)$.

Proof of Result 12: Consider a woman in the period- $T$ cohort of age $x$ and parity $j$ specified in Result 12 . The parity progression ratio to the $j+1$ st child, ${ }_{1} \pi_{j}^{T}\left(x \mid \gamma^{s}, \delta^{s}\right)$, equals the lifetime birth probability for $n=1$ that is given by ${ }_{1} F_{j}^{T}\left(x, \omega \mid \gamma^{s}, \delta^{s}\right)$. For $n>$ 1, i.e., for the transition to parity $j+n$, the parity progression probability ${ }_{n} \pi_{j}^{T}\left(x \mid \gamma^{s}, \delta^{s}\right)$ is the probability of progressing to parity $j+n$ conditional on attaining parity $j+n-1$, and the parity progression probability therefore follows from the lifetime birth probabilities as in Eq. (19). The index of total fertility then follows by calculating the expected number of additional children born to a women in the period- $T$ cohort of age $x$ and parity $j$ from either the period birth lifetime probabilities or period parity progression ratios and adding the parity of the woman in the reference year.

Proof of Result 13: The conditional cohort mean age at birth in Eq. (20) follows from the standard integration of the rate of attaining parity $j+n$ at some age $a$, given by ${ }_{n-1} D_{j}^{T}\left(x, a \mid \gamma^{s}, \delta^{s}\right) \cdot m_{j+n-1}^{s}(a, T+a-x)$, times the age $a$, and dividing by the lifetime probability of attaining a final parity of at least $j+n$. For the special case of $n=$ 1 , the parity distribution ${ }_{0} D_{j}^{T}\left(x, a \mid \gamma^{s}, \delta^{s}\right)$ is the probability of 'surviving' in parity $i$ until age $a$ and can be written in terms of the childbearing intensities experienced by the synthetic cohort as ${ }_{0} D_{j}^{T}\left(x, a \mid \gamma^{s}, \delta^{s}\right)=\exp \left[-\int_{x}^{a} m_{j}^{s}(z, T+z-x) d z\right]$. Integration by parts then yields Hajnal's formula. 
Proof of Result 14: The overall mean age at birth is just the weighted average of the parity-specific mean ages, where weights are given by the lifetime birth probabilities for the respective parities. The denominator $T F_{j}^{T}\left(x \mid \gamma^{s}, \delta^{s}\right)-j=\sum_{k \geq 1}{ }_{k} F_{j}^{T}\left(x, \omega \mid \gamma^{s}, \delta^{s}\right)$ is the expected number of additional births of parity to women in the period- $T$ cohort of age $x$ and parity $j$.

Proof of Result 15: The proof of Result 15 proceeds in four steps. For all steps we consider the period- $T$ cohort of age $a$ and parity $i$ that is specified in Result 15 .

First, we consider the relation between the proportion of women surviving in the childless state in the two postponement scenarios. That is, we consider the relation between ${ }_{0} D_{0}^{T}\left(x, a \mid \gamma^{s}, \delta^{s}=0\right)$ and ${ }_{0} D_{0}^{T}\left(x, a \mid \gamma^{s}=0, \delta^{s}=0\right)$, which can be determined using the childbearing intensities experienced in the two postponement scenarios. If the annual mean changes $\gamma_{0}^{s}$ prevail during the life-course of the period- $T$ cohort of age $x$ and parity zero, this cohort is subject to the first-birth intensities $q_{0}(T)\left(1-\gamma_{0}^{s}\right) \phi_{0}^{T}\left(a-\gamma_{0}^{s} \cdot(a-x)\right)$ when it attains age $a$. These intensities follow directly from Eq. (14) by inserting the specific assumptions about the mean change $\gamma_{0}^{s}$ and the variance change $\delta_{0}^{s}=0$. In the postponement stops scenario, the synthetic cohort at age $a$ is subject to the first birth childbearing intensities $m_{0}^{\prime}(a, T)=q_{0}(T) \phi_{0}^{T}(a)$. We therefore obtain

$$
\begin{aligned}
& { }_{0} D_{0}^{T}\left(x, a \mid \gamma^{s}, \delta^{s}=0\right) \\
= & \exp \left[-q_{0}(T)\left(1-\gamma_{0}^{s}\right) \int_{x}^{a} \phi_{0}^{T}\left(z-\gamma_{0}^{s}(z-x)\right) d z\right] \\
= & \exp \left[-q_{0}(T) \int_{x}^{a-\gamma_{0}^{s} \cdot(a-x)} \phi_{0}^{T}(\zeta) d \zeta\right] \\
= & { }_{0} D_{0}^{T}\left(x, a-\gamma_{0}^{s} \cdot(a-x) \mid \gamma^{s}=0, \delta^{s}=0\right),
\end{aligned}
$$

where the first equality follows from the definition of ${ }_{0} D_{0}^{T}\left(x, a \mid \gamma^{s}, \delta^{s}=0\right)$, the second equality follows by a change of variable from $z$ to $\zeta=z-\gamma^{s} \cdot(z-x)$, and the final equality follows from the definition of ${ }_{0} D_{0}^{T}\left(x, a \mid \gamma^{s}=0, \delta^{s}=0\right)$.

Second, since $\omega$ constitutes the highest feasible age at childbearing, ${ }_{0} D_{0}^{T}\left(x, a \mid \gamma^{s}\right.$, $\left.\delta^{s}=0\right)={ }_{0} D_{0}^{T}\left(x, \omega \mid \gamma^{s}, \delta^{s}=0\right)$ for all ages $a \geq \omega$, i.e., the proportion of women who remain ultimately childless is fixed at the maximum age at childbearing $\omega$. In addition, using Eq. (31) this also implies that ${ }_{0} D_{0}^{T}\left(x, a \mid \gamma^{s}=0, \delta^{s}=0\right)={ }_{0} D_{0}^{T}\left(x, \omega \mid \gamma^{s}, \delta^{s}=0\right)$ for all ages $a \geq \omega-\gamma_{0}^{s} \cdot(\omega-x)$, i.e., the proportion of women who remain ultimately childless in the postponement stops scenario is fixed at an age that is slightly below the maximum age of childbearing (if $\gamma_{0}^{s}>0$ ) and it is equal to the proportion who remain ultimately childless in the postponement continues scenario. 
Third, we consider the integral $\int_{x}^{w}{ }_{0} D_{0}^{T}\left(x, a \mid \gamma^{s}, \delta^{s}=0\right) d a$ and obtain

$$
\begin{aligned}
& \int_{x}^{\omega}{ }_{0} D_{0}^{T}\left(x, a \mid \gamma^{s}, \delta^{s}=0\right) d a \\
= & \int_{x}^{\omega}{ }_{0} D_{0}^{T}\left(x, a-\gamma_{0}^{s} \cdot(a-x) \mid \gamma^{s}=0, \delta^{s}=0\right) d a \\
= & \frac{1}{1-\gamma_{0}^{s}} \int_{x}^{\omega-\gamma_{0}^{s} \cdot(\omega-x)}{ }_{0} D_{0}^{T}\left(x, \alpha \mid \gamma^{s}=0, \delta^{s}=0\right) d \alpha \\
= & \frac{1}{1-\gamma_{0}^{s}}\left[\int_{x}^{\omega}{ }_{0} D_{0}^{T}\left(x, a \mid \gamma^{s}=0, \delta^{s}=0\right) d a-\gamma_{0}^{s} \cdot(\omega-x) .\right. \\
& \left.{ }_{0} D_{0}^{T}\left(x, \omega \mid \gamma^{s}=0, \delta^{s}=0\right)\right],
\end{aligned}
$$

where the first equality follows from Eq. (31), the second equality follows by changing the variable of integration from age $a$ to $\alpha=a-\gamma_{0}^{s} \cdot(a-x)$, and the forth equality uses the fact that the distribution $\left.D_{0,0}^{T}\left(x, \omega \mid \gamma^{s}=0, \delta^{s}=0\right)\right]$ is constant above all ages $a \geq \omega-\gamma_{0}^{s} \cdot(\omega-x)$.

The expression for $\Delta_{1} C M A B_{0}^{T}(x)$ in Eq. (21) then follows as

$$
\begin{aligned}
& \Delta_{1} C M A B_{0}^{T}(x) \\
= & { }_{1} C M A B_{0}^{T}\left(x \mid \gamma^{s}, \delta^{s}=0\right)-{ }_{1} C M A B_{0}^{T}\left(x \mid \gamma^{s}=0, \delta^{s}=0\right) \\
= & \left(1-{ }_{0} D_{0}^{T}\left(x, \omega \mid \gamma^{s}, \delta^{s}\right)\right)^{-1}\left[\int_{x}^{\omega}{ }_{0} D_{0}^{T}\left(x, a \mid \gamma^{s}, \delta^{s}=0\right) d a-\right. \\
& \left.\int_{x}^{\omega}{ }_{0} D_{0}^{T}\left(x, a \mid \gamma^{s}=0, \delta^{s}=0\right) d a\right] \\
= & \gamma_{0}^{s} \cdot\left[\int_{x}{ }_{0} D_{0}^{T}\left(x, a \mid \gamma^{s}=0, \delta^{s}=0\right) d a-(\omega-x)_{0} D_{0}^{T}\left(x, \omega \mid \gamma^{s}=0, \delta^{s}=0\right)\right] / \\
& {\left[\left(1-{ }_{0} D_{0}^{T}\left(x, \omega \mid \gamma^{s}, \delta^{s}\right)\right)\left(1-\gamma_{0}^{s}\right)\right] } \\
= & \frac{\gamma_{0}^{s}}{1-\gamma_{0}^{s}}\left[{ }_{1} C M A B_{0}^{T}\left(x \mid \gamma^{s}=0, \delta^{s}=0\right)-x\right],
\end{aligned}
$$

where the second equality follows from taking the difference between the two conditional cohort mean ages using Hajnal's formula, the third equality follows from Eq. (33) and some further simplifications, and the final equality follows from the calculation of $C M A B_{0,1}^{T}\left(x \mid \gamma^{s}=0, \delta^{s}=0\right)$ using Hajnal's formula.

The expression (22) in Result 15 is merely a transformation of Eq. (21), and Eq. (23) follows by differentiating Eq. (21) using the fact that $\frac{d}{d x}{ }_{1} C M A B_{0}^{T}\left(x \mid \gamma^{s}=0, \delta^{s}=0\right)=$ 0 for cohorts who have not entered childbearing ages. 